\title{
Electroproduction, photoproduction, and inverse electroproduction of pions in the first resonance region
}

\author{
Yurii S. Surovtsev $^{a *}$, Tatiana D. Blokhintseva ${ }^{a \dagger}$, Petr Bydžovskýb ${ }^{b \ddagger}$, Miroslav Nagy ${ }^{c \S}$ \\ ${ }^{a}$ Joint Institute for Nuclear Research, Dubna 141980, Russia \\ ${ }^{b}$ Nuclear Physics Institute, Czech Academy of Sciences, 25068 Řež, Czech Republic \\ ${ }^{c}$ Institute of Physics, Slovak Academy of Sciences, Dúbravská cesta 9, 84511 Bratislava, Slovakia
}

\begin{abstract}
Methods are set forth for determining the hadron electromagnetic structure in the sub- $N \bar{N}$ threshold timelike region of the virtual-photon "mass" and for investigating the nucleon weak structure in the spacelike region from experimental data on the process $\pi N \rightarrow e^{+} e^{-} N$ at low energies. These methods are formulated using the unified description of photoproduction, electroproduction, and inverse electroproduction of pions in the first resonance region in the framework of the dispersionrelation model and on the basis of the model-independent properties of inverse electroproduction. Applications of these methods are also shown.
\end{abstract}

\section{INTRODUCTION}

Processes of meson electroproduction have played an important role in studying the structure and properties of matter (see, e.g. review [1]). In the past few years, however, reactions with production of dileptons in hadron-hadron and hadron-nucleus collisions have drawn much attention [2, 3, 4, 5, 6, 7]. In these reactions virtual photons, which materialize as dileptons (e.g., the $e^{+} e^{-}$pair), carry unique information on properties of matter because the processes in which the particle structure is formed proceed in the timelike region of the "mass" $\left(\lambda^{2}\right)$ of the virtual photon. Therefore, further investigation of these reactions is necessary and promising in acquisition of new and perhaps unexpected information about the form factors of hadrons and nuclei.

The inverse pion electroproduction (IPE), $\pi N \rightarrow e^{+} e^{-} N$, (or dileptonproduction), being for a long time the only source of information on the nucleon electromagnetic structure in the timelike region, has been investigated both theoretically 8, 9, 10, 11, 12, 13, 14, 15, 16, 17, 18] and experimentally [19, 20, 21] since the beginning of the 1960s. In Refs. 9, 11, 18], we worked out the method of extracting the pion and nucleon electromagnetic form factors from IPE at low energies. This method has been successfully applied in the analysis of experimental data on the nucleon and ${ }^{12} \mathrm{C}$ and ${ }^{7} \mathrm{Li}$ nuclei 20,21 and values of the form factors were obtained for the first time in the timelike region of $\lambda^{2}$ ranging from 0.05 to $0.22(\mathrm{GeV} / \mathrm{c})^{2}$. In Refs. 2, 10], the authors proposed to use IPE at intermediate (above $\pi N$ resonances) energies and small $|t|$ to study the nucleon electromagnetic structure and justified it up to $\lambda^{2} \approx m_{\rho}^{2}$.

Though experimental data 22] on the $p \bar{p} \rightarrow e^{+} e^{-}$process are now available, there still remains a wide range of $\lambda^{2}$ (up to $4 m^{2}$ ), where the form factors cannot be measured directly in these experiments. On the other hand, the intense pion beams available now enable one to perform more detailed experiments on IPE aimed at both extracting the hadron structure and carrying out a multipole analysis similar to those for photoproduction and electroproduction (see,e.g., 23] $)$. Such experiments can address interesting topics. For example, in the $\Delta(1232)\left(P_{33}\right)$ region it is challenging to verify the $\lambda^{2}$ dependence of the color-magnetic-force contribution found in the constituent quark model [24]. It is, therefore, worth recalling the earlier discovered properties of the photoproduction, electroproduction, and IPE to consistently substantiate methods of studying the electromagnetic and weak structure of the nucleon on the basis of the IPE data in the first resonance region, and to provide new results of this analysis.

Additional motive for studying IPE in the first resonance region is the possibility of investigating the nucleon weak structure by utilizing the same data as for the electromagnetic structure. This possibility is based on the current algebra (CA) description and on the remarkable property of IPE. In the IPE process the creation of the $e^{+} e^{-}$pairs of maximal mass (at the "quasithreshold") is dominated by the Born mechanism, whereas the rescattering-effect contributions are at the level of radiative corrections up to the total $\pi N$ energy $w \approx 1.5 \mathrm{GeV}$ (the "quasithreshold theorem") 11. Due to this property, the threshold CA theorems for the pion electroproduction and photoproduction can be justified in the case of IPE up to the indicated energy [12, 15. This allows one to avoid threshold difficulties when using the IPE data (unlike the electroproduction one) for extracting the weak form factors of the nucleon. Furthermore, in the case of IPE there is no strong kinematic restriction inherent to the $\mu$ capture and no kinematic suppression of

\footnotetext{
* E-mail address: surovcev@thsun1.jinr.ru

$\dagger$ E-mail address: blokhin@main1.jinr.ru

‡ E-mail address: bydz@ujf.cas.cz

$\S$ E-mail address: fyzinami@savba.sk
} 
contributions of the induced pseudoscalar nucleon form factor to the cross sections of "straight" processes, such as $\nu N \rightarrow l N$, present because of multiplying by the lepton masses. Information on the pseudoscalar nucleon form factor $G_{P}$, which is practically absent for the above reasons, is important because $G_{P}$ contains contributions of states with the pion quantum numbers and, therefore, it is related to the chiral symmetry breaking. This would enable us, e.g., to test the Goldberger-Treiman relation.

Another aim of this paper is to draw attention of experimenters to the process $\pi N \rightarrow e^{+} e^{-} N$ as a natural and unique laboratory for investigating the hadron structure. One could use these processes for determining the baryon resonance dynamics based on the study of the $\rho^{0}-\omega$ interference pattern [4, 6$]$. On the other hand, investigation of the exclusive reactions $\pi N \rightarrow l^{+} l^{-} N$ in experiments with high-energy pions at large invariant mass of the dilepton and small squared momentum transfer to the nucleon could provide access to generalized parton distributions as suggested in [5].

This paper is organized as follows. In Sec. II we give the basic formalism for the unified treatment of the reactions $\gamma+N \rightarrow \pi+N, e+N \rightarrow e+\pi+N$, and $\pi+N \rightarrow e^{+}+e^{-}+N$. In Sec. III we present our dispersion-relation model for unified description of these three reactions and compare the calculated results with experimental data. To clearly explain our method, we choose a simple version of the model, which satisfactorily describes the data on the photoproduction and electroproduction. In Sec. IV, we outline the method of determining the nucleon electromagnetic form factors from low-energy IPE and discuss some results of its application to analysis of the IPE data on the nucleon. Section V is devoted to extracting the pseudoscalar nucleon form factor from the same IPE data, and interpretation of the results is given. Concluding remarks are presented in Sec. VI. Appendices present the relations between the amplitudes, derivation of the quasithreshold constraints for the multipole amplitudes on the basis of the principle of the first-class maximum analyticity, and explanation of the compensation effect.

\section{BASIC FORMALISM}

We consider the reactions $\gamma+N \rightarrow \pi+N, e+N \rightarrow e+\pi+N$, and $\pi+N \rightarrow e^{+}+e^{-}+N$ in the framework of the unified model. This approach is natural because in the one-photon approximation, due to the $T$-invariance, research into these three processes is related to a study of the process $\gamma^{*} N \leftrightarrow \pi N$ with the hadron current $J_{\mu}\left(s, t, \lambda^{2}\right)$, where $\lambda^{2}=0,<0$, and $>0$ correspond to the pion photoproduction, electroproduction, and IPE, respectively. This allows us to predict peculiarities of the IPE dynamics on the basis of a rich experimental material on the electroproduction and photoproduction and to test the reliability of the unified model for these three processes.

The $S$-matrix element for the electroproduction in the one-photon approximation is

$$
S_{f i}=\delta_{f i}+i(2 \pi)^{4} \delta\left(p_{1}+k-p_{2}-q\right) \frac{e m}{\sqrt{2 q_{0} p_{10} p_{20} k_{10} k_{20}}} \frac{m_{e}^{2}}{\lambda^{2}} \varepsilon^{\mu} J_{\mu}\left(s, t, \lambda^{2}\right),
$$

where

$$
\varepsilon^{\mu}=\bar{u}\left(k_{2}\right) \gamma^{\mu} u\left(k_{1}\right)
$$

is the matrix element of the lepton electromagnetic current, $k_{1}=\left(k_{10}, \vec{k}_{1}\right)\left(k_{2}\right), p_{1}\left(p_{2}\right)$, and $q$ are the four-momenta of the initial (final) electron, nucleon, and final pion, respectively. Momentum of the virtual photon is $k=k_{1}-k_{2}$ $\left(k=k_{1}+k_{2}\right.$ for IPE), $k^{2}=k_{0}^{2}-\vec{k}^{2} \equiv \lambda^{2}$, and $s=\left(p_{1}+k\right)^{2}$ and $t=(k-q)^{2}$ are Mandelstam variables. Conservation of the lepton and hadron electromagnetic currents implies $J_{\mu} k^{\mu}=\varepsilon_{\mu} k^{\mu}=0$.

Assuming the $T$-invariance, for the IPE process one must use the spinor $v\left(k_{1}\right)$ instead of $u\left(k_{1}\right)$ in the lepton current (22). Then $k$ is timelike, and $4 m_{e}^{2} \leq \lambda^{2} \leq(\sqrt{s}-m)^{2}$ is the range of $\lambda^{2}$ values for fixed $s$.

The hadron current $J_{\mu}\left(s, t, \lambda^{2}\right)$ can be expanded using the six independent covariant gauge-invariant structures $M_{i}$ [9, 25]:

$$
J_{\mu} \varepsilon^{\mu}=\sum_{i=1}^{6} A_{i}\left(s, t, \lambda^{2}\right) \bar{u}\left(p_{2}\right) M_{i} u\left(p_{1}\right),
$$

where

$$
\begin{array}{ll}
M_{1}=\frac{i}{2} \gamma_{5} \gamma^{\mu} \gamma^{\nu} F_{\mu \nu}, & M_{4}=2 i \gamma_{5} \gamma^{\mu} P^{\nu} F_{\mu \nu}-2 m M_{1} \\
M_{2}=-2 i \gamma_{5} P^{\mu} q^{\nu} F_{\mu \nu}, & M_{5}=-i \gamma_{5} k^{\mu} q^{\nu} F_{\mu \nu} \\
M_{3}=i \gamma_{5} \gamma^{\mu} q^{\nu} F_{\mu \nu}, & M_{6}=i \gamma_{5} k^{\mu} \gamma^{\nu} F_{\mu \nu}
\end{array}
$$


with $F_{\mu \nu}=\varepsilon_{\mu} k_{\nu}-k_{\mu} \varepsilon_{\nu}$ and $P=\frac{1}{2}\left(p_{1}+p_{2}\right)$. The invariant amplitudes $A_{i}\left(s, t, \lambda^{2}\right)(i=1, \cdots, 6)$ are free from kinematic constraints, but $A_{2}$ and $A_{5}$ have a kinematic pole at $t=m_{\pi}^{2}+\lambda^{2}$. The amplitudes $A_{5}$ and $A_{6}$ are absent in the photoproduction.

The matrix element (3) can be expressed through the scalar c.m. amplitudes

$$
\frac{m_{e}^{2}}{\lambda^{2}} \varepsilon^{\mu} J_{\mu}=\chi_{2}^{+} F \chi_{1}
$$

where $\chi_{1}$ and $\chi_{2}$ are the Pauli spinors, and

$$
\begin{aligned}
F=\quad & i(\vec{\sigma} \cdot \vec{\varepsilon}-\vec{\sigma} \cdot \tilde{\vec{k}} \tilde{\vec{k}} \cdot \vec{\varepsilon}) F_{1}+\vec{\sigma} \cdot \tilde{\vec{q}} \vec{\sigma} \cdot[\tilde{\vec{k}} \times \vec{\varepsilon}] F_{2}+i \vec{\sigma} \cdot \tilde{\vec{k}}(\tilde{\vec{q}} \cdot \vec{\varepsilon}-\tilde{\vec{q}} \cdot \tilde{\vec{k}} \tilde{\vec{k}} \cdot \vec{\varepsilon}) F_{3}+ \\
& +i \vec{\sigma} \cdot \tilde{\vec{q}}(\tilde{\vec{q}} \cdot \vec{\varepsilon}-\tilde{\vec{q}} \cdot \tilde{\vec{k}} \tilde{\vec{k}} \cdot \vec{\varepsilon}) F_{4}+i \frac{k^{2}}{k_{0}} \vec{\sigma} \cdot \tilde{\vec{k}} \tilde{\vec{k}} \cdot \vec{\varepsilon} F_{5}+i \frac{k^{2}}{k_{0}} \vec{\sigma} \cdot \tilde{\vec{q}} \tilde{\vec{k}} \cdot \vec{\varepsilon} F_{6} \cdot
\end{aligned}
$$

Here $\tilde{\vec{q}}$ and $\tilde{\vec{k}}$ are unit vectors. The amplitudes $F_{1,2,3,4}$ describe the process with the transversal photons and $F_{5,6}$ with the longitudinal ones. The relations between the amplitudes $F_{i}$ and $A_{i}$ are listed in Appendix A.

In the isotopic space $F_{i}$ (and $\left.A_{i}\right)$ are matrices

$$
F_{i}^{\alpha}=\tau_{\alpha} F_{i}^{(0)}+\delta_{\alpha 3} F_{i}^{(+)} \pm \frac{1}{2}\left[\tau_{\alpha}, \tau_{3}\right] F_{i}^{(-)}
$$

where the upper sign corresponds to $\gamma^{*} N \rightarrow \pi N$ and the lower one to $\pi N \rightarrow \gamma^{*} N$ process.

For the physical processes, we have

$$
\begin{aligned}
& F_{i}\left(\gamma^{*} p \leftrightarrow \pi^{+} n\right)=\sqrt{2}\left(F_{i}^{(0)}+F_{i}^{(-)}\right), F_{i}\left(\gamma^{*} n \leftrightarrow \pi^{-} p\right)=\sqrt{2}\left(F_{i}^{(0)}-F_{i}^{(-)}\right), \\
& F_{i}\left(\gamma^{*} p \leftrightarrow \pi^{0} p\right)=F_{i}^{(0)}+F_{i}^{(+)}, \quad F_{i}\left(\gamma^{*} n \leftrightarrow \pi^{0} n\right)=-F_{i}^{(0)}+F_{i}^{(+)} .
\end{aligned}
$$

The differential cross section for the electroproduction is written down in the following form taking into account a possibility of the longitudinal polarization of the electron

$$
\begin{aligned}
\frac{d^{3} \sigma}{d \Omega_{e}^{L} d k_{20}^{L} d \Omega_{\pi}}= & \frac{\alpha}{2 \pi^{2}} \frac{k_{20}^{L}}{k_{10}^{L}} \frac{\mathbf{k}_{L}}{\left(-\lambda^{2}\right)} \frac{1}{1-\epsilon} \frac{\mathbf{q}}{\mathbf{k}} \frac{1}{4}\left[T_{1}+\epsilon \cos 2 \phi T_{2}+\right. \\
& \left.\sqrt{2 \epsilon(1+\epsilon)} \cos \phi \sqrt{-\lambda^{2}} T_{3}-\epsilon \lambda^{2} T_{4}-\xi \sqrt{2 \epsilon(1-\epsilon)} \sin \phi \sqrt{-\lambda^{2}} T_{5}\right]
\end{aligned}
$$

where $\epsilon=\left[1-2\left(\mathbf{k}_{L}^{2} / \lambda^{2}\right) \tan ^{2}\left(\theta_{L} / 2\right)\right]^{-1}, \mathbf{k} \equiv|\vec{k}|$ and $\mathbf{q}$ are magnitudes of the photon and pion c.m. momenta, respectively; $\phi$ is the azimuthal angle between planes of the electron scattering and the reaction $\gamma^{*} N \rightarrow \pi N ; \mathbf{k}_{L}, k_{10}^{L}$ $\left(k_{20}^{L}\right)$ and $\theta_{L}$ are the photon-momentum magnitude, the initial (final) energy and the electron scattering angle in lab frame, respectively; and $\Omega_{e}^{L}$ and $\Omega_{\pi}$ are the solid angles of the scattered electron in lab and pion in c.m. of the $\pi N$ system, respectively. Degree of the longitudinal polarization of the electron is $\xi=\vec{l} \cdot \vec{k}_{1} / \mathbf{k}_{\mathbf{1}}$ where $\vec{l}$ is the polarization vector of the electron in its rest system.

The IPE differential cross section reads

$$
\begin{aligned}
\frac{d^{3} \sigma}{d \Omega^{\gamma} d \Omega^{e} d k^{2}}= & \frac{\alpha}{16 \pi^{2}} \frac{1}{\lambda^{2}} \frac{\mathbf{k}}{\mathbf{q}} \frac{1}{4}\left[\left(1+\cos ^{2} \theta\right) T_{1}-\sin ^{2} \theta \cos 2 \phi T_{2}+\right. \\
& \left.\lambda \sin 2 \theta \cos \phi T_{3}+\lambda^{2} \sin ^{2} \theta T_{4}+\xi \lambda \sin \theta \sin \phi T_{5}\right],
\end{aligned}
$$

where $\theta$ is the angle between the momenta of the final nucleon and electron in the $e^{+} e^{-}$c.m. system, $\phi$ is the angle between planes of the reaction $\pi N \rightarrow \gamma^{*} N$ and the $e^{+} e^{-}$pair production, $\Omega^{\gamma}$ is the solid angle of the virtual photon production in the c.m. of the $\pi N$ system, and $\Omega^{e}$ is the solid angle of electron in the c.m. of the $e^{+} e^{-}$pair. $T_{1}$ in formulas (8) and (9) describes the processes $\gamma^{*} N \leftrightarrow \pi N$ with the unpolarized transversal photons, $T_{2}$ characterizes the asymmetry of contributions of the transversal virtual photons linearly polarized in the plane of the $\gamma^{*} N \leftrightarrow \pi N$ and normally to it, $T_{4}$ is the contribution of the longitudinal photons, and $T_{3}$ and $T_{5}$ are the real and imaginary parts of the interference contribution of transversal and longitudinal photon in the helicity basis. It is seen that $T_{5}$ is related with the contribution of longitudinal polarization of electron to the cross section. The differential cross sections (8) and (9), measured for the processes with electron polarized in one direction, $\sigma(\xi=+1)$, and in the opposite one, $\sigma(\xi=-1)$, generally should display the asymmetry $P_{e}$ given by the contribution of $T_{5}$

$$
P_{e}=\frac{\sigma(+1)-\sigma(-1)}{\sigma(+1)+\sigma(-1)} .
$$


The quantities $T_{i}$ are related to the amplitudes $F_{i}$ in the c.m. frame as

$$
\begin{aligned}
& T_{1}=\frac{\alpha m^{2}}{\pi s}\left[\left|F_{1}\right|^{2}+\left|F_{2}\right|^{2}-2 \cos \theta^{\gamma} \operatorname{Re}\left(F_{1} F_{2}^{*}\right)\right]+T_{2}, \\
& T_{2}=\frac{\alpha m^{2}}{2 \pi s} \sin ^{2} \theta^{\gamma}\left[\left|F_{3}\right|^{2}+\left|F_{4}\right|^{2}-2 \operatorname{Re}\left(F_{1} F_{4}^{*}+F_{2} F_{3}^{*}+\cos \theta^{\gamma} F_{3} F_{4}^{*}\right)\right], \\
& T_{3}=\frac{\alpha m^{2}}{\pi s} \sin \theta^{\gamma} \operatorname{Re}\left[\left(F_{2}+F_{3}+\cos \theta^{\gamma} F_{4}\right) F_{5}^{*}+\left(F_{1}+\cos \theta^{\gamma} F_{3}+F_{4}\right) F_{6}^{*}\right], \\
& T_{4}=\frac{\alpha m^{2}}{\pi s}\left[\left|F_{5}\right|^{2}+\left|F_{6}\right|^{2}+2 \cos \theta^{\gamma} \operatorname{Re}\left(F_{5} F_{6}^{*}\right)\right], \\
& T_{5}=2 \frac{\alpha m^{2}}{\pi s} \sin \theta^{\gamma} \operatorname{Im}\left[\left(F_{2}^{*}+F_{3}^{*}+\cos \theta^{\gamma} F_{4}^{*}\right) F_{5}+\left(F_{1}^{*}+\cos \theta^{\gamma} F_{3}^{*}+F_{4}^{*}\right) F_{6}\right] .
\end{aligned}
$$

\section{DISPERSION-RELATION MODEL}

To obtain reliable information on the nucleon structure, it is important to find kinematic conditions under which the IPE dynamics is determined mainly by a model-independent part of interactions, the Born one. To this end, we utilize such general principles, as analyticity, unitarity, and Lorentz invariance and the phenomenology of the processes $e N \rightarrow e \pi^{ \pm} N$ and $\gamma N \leftrightarrow \pi^{ \pm} N$, considered in the framework of the unified (including IPE) model. We use a simple version of the model, which describes the experimental data satisfactorily with a minimal number of parameters (electromagnetic form factors). This allows one to carry out a simple analytic continuation from the spacelike to timelike values of $\lambda^{2}$. For isovector amplitudes, the fixed- $t$ dispersion relations without subtractions at finite energy are used, with the spectral functions describing the magnetic excitation of the $P_{33}(1232)$ resonance. The isoscalar amplitudes are described only by the Born terms since the $P_{33}(1232)$ resonance does not contribute to them due to the isospin conservation. In Refs. [9, 25], it was shown that this model is successful in combined description of experimental data on the pion electroproduction, photoproduction, and IPE in the total-energy region from the threshold up to $w=\sqrt{s} \approx 1.5 \mathrm{GeV}$. Moreover, this model is adequate for our purpose, namely to formulate the method for determination of the form factors in the timelike region, for the following reasons. First, in the dispersion-relation approach, the spectral functions in the first resonance region are expressed through the nucleon electromagnetic form factors and the phase shift of the $\pi N$-scattering $\delta_{33}(w)$. This reduces considerably the number of fitted parameters, which is especially important in the IPE analysis. Second, in the $P_{33}(1232)$ region, the electric $E_{1+}$ and scalar $S_{1+}$ quadrupoles, which (as the magnetic dipole $M_{1+}$ ) describe excitation of the resonance $P_{33}(1232)$, amount to not more than $15 \%$ of $M_{1+}$. For example, the photoproduction data 26 ] give the value $(-2.5 \pm 0.1 \pm 0.2) \%$ for the $E_{1+} / M_{1+}$ ratio. Phenomenological results for the $\lambda^{2}$ dependence of the $E_{1+} / M_{1+}$ and $S_{1+} / M_{1+}$ ratios are not stable yet and depend upon the method of their extraction from electroproduction data (see discussion of this point in Ref. 27], p. 698). However, these ratios do not exceed respectively, 7 and $15 \%$ up to $\lambda^{2}=-4(\mathrm{GeV} / \mathrm{c})^{2}$. Based on the quark model calculations (see, e.g., Refs. 24, 28]), we suppose that an analogous situation takes place also for $\lambda^{2}>0$, at least up to $\lambda^{2} \approx 0.3(\mathrm{GeV} / \mathrm{c})^{2}$. Therefore, at the first stage of our analysis we neglect the quadrupole excitations of $P_{33}(1232)$, which we expect to be a good approximation for processes with unpolarised nucleons.

The conventional procedure of Reggeization provides us with behaviour of the invariant amplitudes for $s \rightarrow \infty$ and small $|t|[29]$

$$
A_{i} \sim s^{\alpha(t)-1}(i \neq 5), \quad A_{5} \sim s^{\alpha(t)}
$$

Consequently, in a complete $s$-channel description, we should write a fixed- $t$ dispersion relation with one subtraction at finite energy for the isovector amplitude $A_{5}^{(-)}$and without subtractions for the remaining amplitudes, taking into account their crossing properties. However, the dispersion integrals with the spectral functions which describe the magnetic excitation of the $P_{33}(1232)$ resonance converge very well already at $\approx 2 \mathrm{GeV}$ for all the amplitudes $A_{i}$. Therefore, we use the fixed- $t$ dispersion relations without subtraction at the finite energy [9, 25] for all isovector amplitudes

$$
\begin{aligned}
& A_{i}^{( \pm)}\left(s, t, \lambda^{2}\right)=\tilde{R}_{5}^{(-)}+c_{5}+R_{i}^{( \pm)}\left(\frac{1}{m^{2}-s} \pm \frac{\epsilon_{i}}{m^{2}-u}\right)+ \\
& \quad+\frac{1}{\pi} \int_{\left(m+m_{\pi}\right)^{2}}^{\infty} d s^{\prime} \operatorname{Im} A_{i}^{( \pm)}\left(s^{\prime}, t, \lambda^{2}\right)\left(\frac{1}{s^{\prime}-s-i \varepsilon} \pm \frac{\epsilon_{i}}{s^{\prime}-u}\right)
\end{aligned}
$$


and we take the Born approximation for the isoscalar ones

$$
A_{i}^{(0)}\left(s, t, \lambda^{2}\right)=R_{i}^{(0)}\left(\frac{1}{m^{2}-s}+\frac{\epsilon_{i}}{m^{2}-u}\right),
$$

where $\epsilon_{1,2,4}=-\epsilon_{3,5,6}=1, \quad u=2 m^{2}+m_{\pi}^{2}+\lambda^{2}-s-t$,

$$
\begin{aligned}
& R_{1}^{( \pm, 0)}=-\frac{g}{2} F_{1}^{v, s}\left(\lambda^{2}\right), \quad R_{2}^{( \pm, 0)}=\frac{g F_{1}^{v, s}\left(\lambda^{2}\right)}{t-m_{\pi}^{2}-\lambda^{2}}, \\
& R_{3}^{( \pm, 0)}=R_{4}^{( \pm, 0)}=(-,+) \frac{g}{2} F_{2}^{v, s}\left(\lambda^{2}\right), \quad R_{5}^{( \pm, 0)}=R_{6}^{( \pm, 0)}=0 \\
& \tilde{R}_{5}^{(-)}=\frac{2 g}{\lambda^{2}}\left[\frac{F_{1}^{v}\left(\lambda^{2}\right)}{t-m_{\pi}^{2}-\lambda^{2}}-\frac{F_{\pi}\left(\lambda^{2}\right)}{t-m_{\pi}^{2}}\right]
\end{aligned}
$$

and

$$
c_{5}=\frac{2}{m_{\pi}^{2}+\lambda^{2}-t} \frac{1}{\pi} \int_{\left(m+m_{\pi}\right)^{2}}^{\infty} \frac{d s^{\prime}}{s^{\prime}-m^{2}} \lim _{t \rightarrow m_{\pi}^{2}+\lambda^{2}}\left[\left(t-m_{\pi}^{2}-\lambda^{2}\right) \operatorname{Im} A_{5}^{(-)}\left(s^{\prime}, t, \lambda^{2}\right)\right],
$$

with the $\pi N$ coupling constant $g^{2} / 4 \pi=14.6$ and the normalisation of the form factors: $F_{1}^{v, s}(0)=F_{\pi}(0)=$ $1,2 m F_{2}^{v}(0)=3.7,2 m F_{2}^{s}(0)=-0.12$. The terms $\tilde{R}_{5}^{(-)}$and $c_{5}$ belong only to the amplitude $A_{5}^{(-)}$.

Note that $A_{2}$ and $A_{5}$ have the kinematic pole at $t=m_{\pi}^{2}+\lambda^{2}$. However, these singularities are cancelled out kinematically because these amplitudes enter into the matrix element through the combination $\left(s-m^{2}\right) A_{2}+\lambda^{2} A_{5}$ which is equal to $2 B_{3}-B_{2}$, where $B_{2}$ and $B_{3}$ are the Ball amplitudes which have been proved to have no kinematic singularities [25]. In specific model calculations, the condition

$$
\lim _{t \rightarrow m_{\pi}^{2}+\lambda^{2}}\left(t-m_{\pi}^{2}-\lambda^{2}\right)\left[\left(s-m^{2}\right) A_{2}+\lambda^{2} A_{5}\right]=0,
$$

which is ensured by the form of the term $c_{5}$ (15), guarantees absence of the singularity at $t=m_{\pi}^{2}+\lambda^{2}$.

The spectral functions $\operatorname{Im} A_{i}^{( \pm)}\left(s^{\prime}, t, \lambda^{2}\right)$ are supposed to describe the magnetic excitation of the $P_{33}(1232)$ resonance

$$
\operatorname{Im} A_{i}^{( \pm)}\left(s, t, \lambda^{2}\right)=\frac{4 \pi}{3}\left(\begin{array}{c}
2 \\
-1
\end{array}\right) \frac{m G_{M}^{v}\left(\lambda^{2}\right) \sin ^{2} \delta_{33}(w)}{w g \mathbf{q}^{3}\left[(w+m)^{2}-\lambda^{2}\right]} a_{i}\left(w, t, \lambda^{2}\right)
$$

where $G_{M}^{v}=F_{1}^{v}+2 m F_{2}^{v}, \quad \delta_{33}(w)$ is the corresponding phase shift of the $\pi N$-scattering amplitude, for which we utilize the prescription from Ref. [30], and

$$
\begin{gathered}
a_{i}\left(w, t, \lambda^{2}\right)=\alpha_{i}(w, t)-\lambda^{2} \beta_{i}(w), \quad a_{2,5}\left(w, t, \lambda^{2}\right)=\frac{\alpha_{2,5}(w, t)-\lambda^{2} \beta_{2,5}(w)}{t-m_{\pi}^{2}-\lambda^{2}}, \\
\quad i=1,3,4,6
\end{gathered}
$$

with the coefficients $\alpha_{i}, \beta_{i}$

$$
\begin{array}{ll}
\alpha_{1}=\frac{1}{2}(w+m)\left[(w+m) q_{0}-m_{\pi}^{2}+3 t\right], & \beta_{1}=\frac{1}{2}(w+m- \\
\alpha_{2}=\frac{3}{2}(w+m)\left(m_{\pi}^{2}-t\right), & \beta_{2}=\frac{1}{2}(w+m)+ \\
\alpha_{3}=-\frac{1}{2}(w+m)\left(w+m-q_{0}\right)-\frac{3}{4}\left(m_{\pi}^{2}-t\right), & \beta_{3}=\frac{3}{4}, \\
\alpha_{4}=(w+m)\left(w+m+\frac{1}{2} q_{0}\right)-\frac{3}{4}\left(m_{\pi}^{2}-t\right), & \beta_{4}=\frac{3}{4}, \\
\alpha_{5}=2\left(s-m^{2}\right)\left(w+m+\frac{1}{2} q_{0}\right)-\frac{3}{2}(w-m)\left(m_{\pi}^{2}-t\right), & \beta_{5}=\frac{3}{2}(w-m), \\
\alpha_{6}=-\frac{1}{2}(w+m) q_{0}-\frac{1}{4}\left(m_{\pi}^{2}+3 t\right), & \beta_{6}=-\frac{3}{4} .
\end{array}
$$

Furthermore, the results of the photoproduction multipole analyses [23] allow us to set $E_{0+}^{(0)}=0$ above the $P_{33}(1232)$ energy. Prescriptions for the pion and nucleon electromagnetic form factors are taken from Refs. 31, 32]. The model described above is the first (simple) reliable version of the more complex model for unified treatment of contemporary experimental data on the pion photoproduction, electroproduction, and IPE in the energy region which spans from the threshold up to the second $\pi N$ resonance.

In Fig. 1 we compare results of our model for the differential cross section with $\pi^{-}$-photoproduction data. In Fig. 2 we show comparison with the electroproduction data on the proton for the transversal $\sigma_{T}$ and longitudinal $\sigma_{L}$ parts of the cross section

$$
\frac{d^{2} \sigma}{d \Omega_{e}^{L} d k_{20}^{L}}=\frac{\alpha}{2 \pi^{2}} \frac{s-m^{2}}{2 m\left(-\lambda^{2}\right)} \frac{k_{20}^{L}}{k_{10}^{L}} \frac{1}{1-\epsilon}\left[\sigma_{T}\left(w, \lambda^{2}\right)+\epsilon \sigma_{L}\left(w, \lambda^{2}\right)\right]
$$



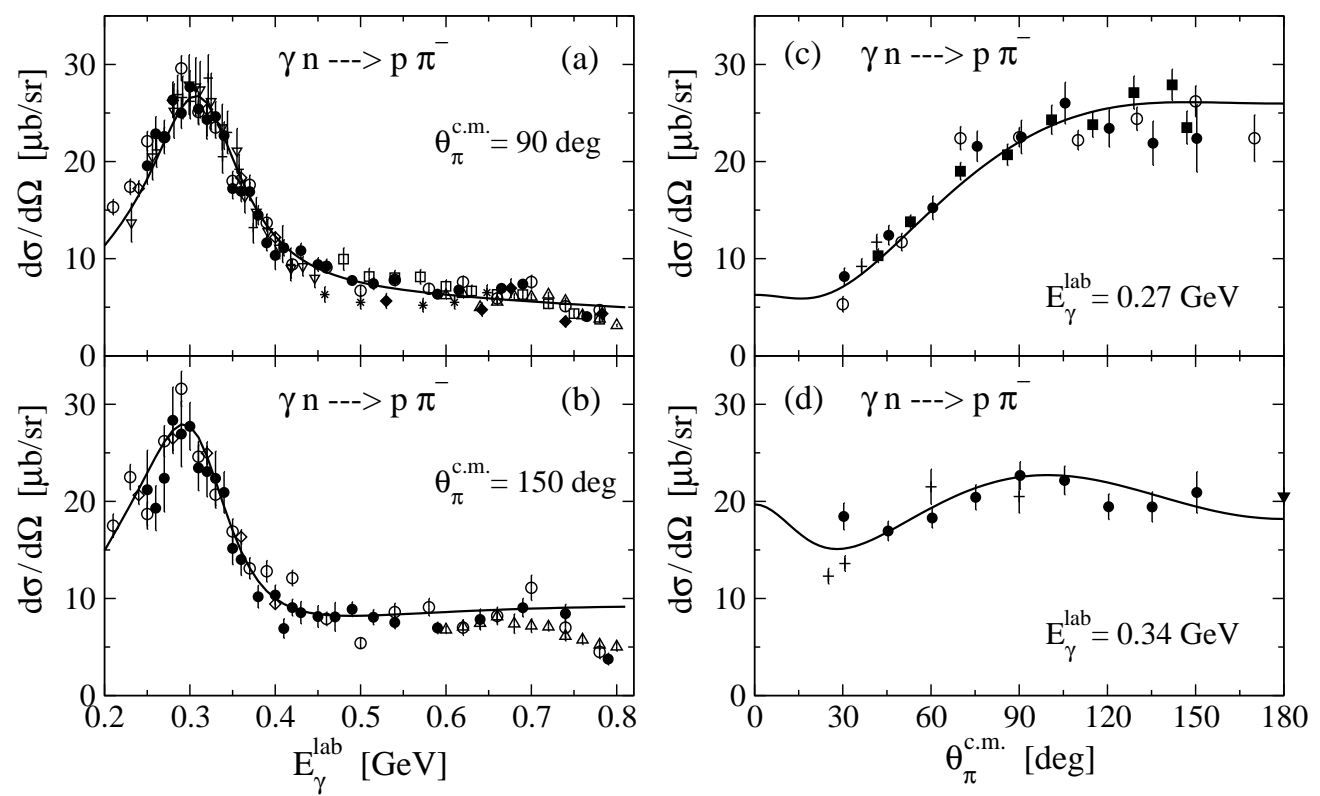

FIG. 1: Differential cross section for $\gamma n \rightarrow p \pi^{-}$as a function of energy in (a) and (b) and pion angle in (c) and (d). Experimental data are taken from Ref. [33].
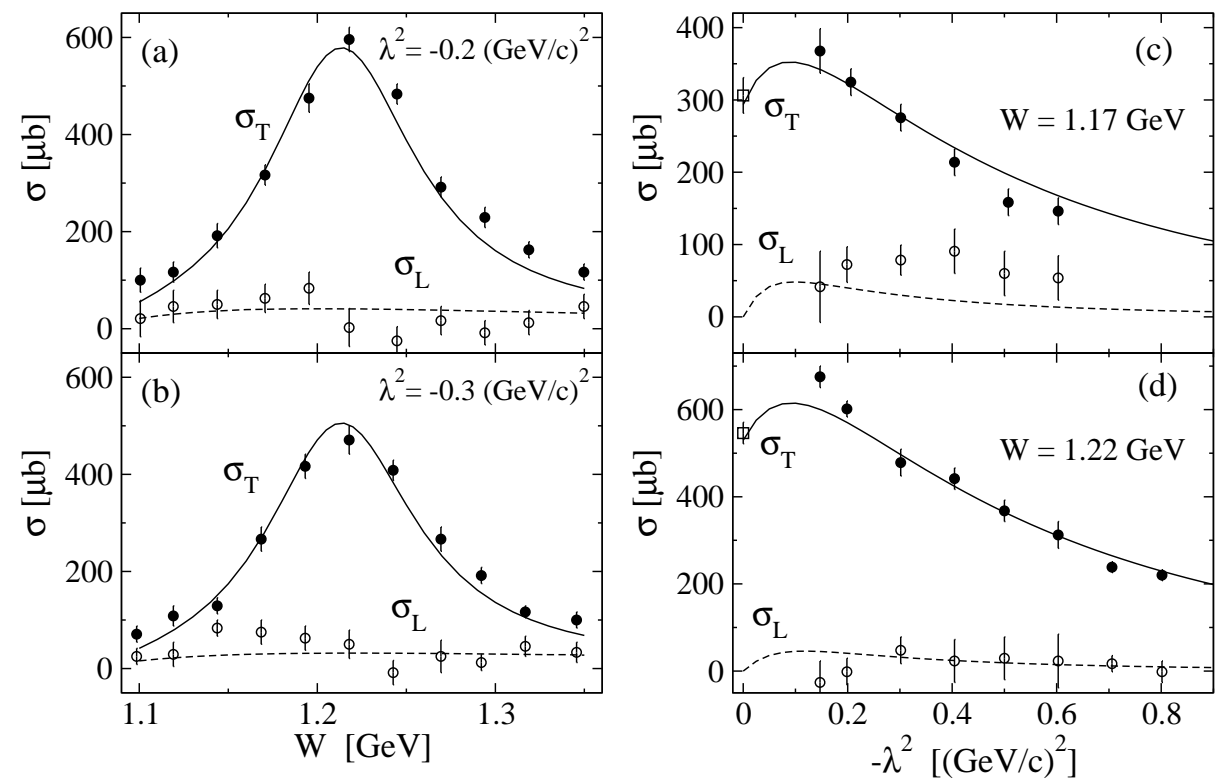

FIG. 2: $W$ and $\lambda^{2}$ dependences of the transversal $\sigma_{T}$ and longitudinal $\sigma_{L}$ parts of the cross section (20) for the inclusive pion $\left(\pi^{+}\right.$and $\left.\pi^{0}\right)$ electroproduction on the proton in the first resonance region. Experimental data on the inclusive $p\left(e, e^{\prime}\right)$ process are from Ref. [34] and the photoproduction points, squares in (c) and (d), are from Ref. [35].

The differential cross sections for the forward electroproduction of pions by transversal and longitudinal virtual photons are presented in Fig. [3]

In general, we obtain quite a good agreement with the pion photoproduction and electroproduction data on unpolarized nucleons, especially in the region of the $P_{33}(1232)$ resonance $\left(E_{\gamma}^{l a b} \doteq 0.34 \mathrm{GeV}\right)$. In the case of the $\pi^{-}$ photoproduction, the results are very good up to $w \approx 1.5 \mathrm{GeV}\left(E_{\gamma}^{l a b} \approx 0.73 \mathrm{GeV}\right)$, see Fig. [1]

It is obvious that our model can be further improved by including the quadrupole excitation of the $P_{33}(1232)$ resonance $\left(E_{1+}^{ \pm}\right.$and $\left.L_{1+}^{ \pm}\right)$in the spectral functions. However, a still more elaborate model should include, in addition to 


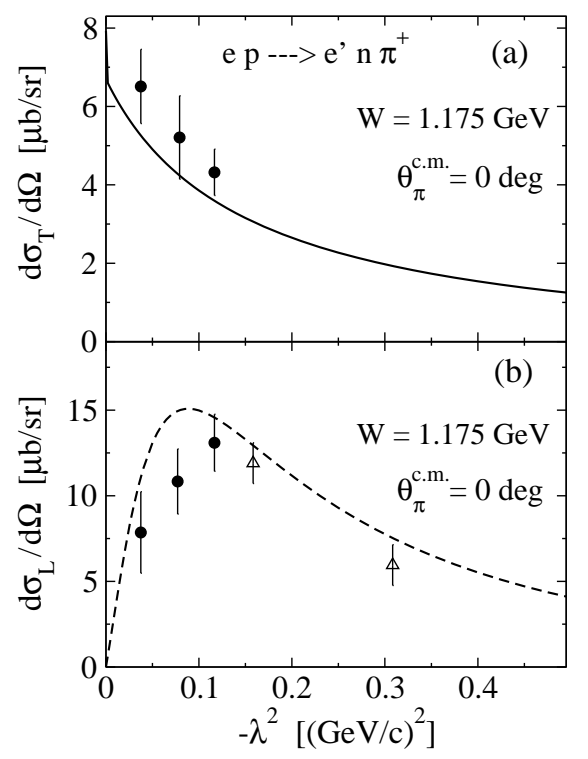

FIG. 3: $\lambda^{2}$ dependence of the differential cross section for the forward electroproduction of pions by transversal (a) and longitudinal (b) virtual photons. Experimental data are from Refs. [36] and [37].

the quadrupole excitation, contributions of other nucleon isobars and high-energy "tails" to the absorption parts of the amplitudes to ensure a balanced consideration of small corrections. Furthermore, analytic continuation of the corrected absorption parts of the amplitudes into the unobservable region in the dispersion integrals, $\left(m+m_{\pi}\right)^{2} \leq s \leq(m+\lambda)^{2}$ for $\lambda^{2}>m_{\pi}^{2}$, requires use of the quasithreshold relations (following from causality analyticity) between the electric and longitudinal multipoles [29] in which "toroid" multipoles appear [38]. On the contrary, the analytic continuation with the approximation (17) is immediate. However, having in mind the quality of contemporary experimental data, the above-stated simple model seems to be quite sufficient (see Figs. 113).

\section{METHOD OF DETERMINING THE NUCLEON ELECTROMAGNETIC STRUCTURE FROM THE LOW-ENERGY IPE}

Application of the model to the calculations for IPE shows an interesting growth of the relative contribution of the Born terms with $\lambda^{2}$ and their dominance in the neighbourhood of the value $\lambda^{2}=(\sqrt{s}-m)^{2}[9]$. This approximate dominance of the Born terms has a model-independent explanation. It is related to the quasithreshold theorem [11], which means that at the quasithreshold, $\mathbf{k} \rightarrow 0, \lambda^{2} \rightarrow \lambda_{\max }^{2}=(\sqrt{s}-m)^{2}$, the IPE amplitude becomes the Born one in the energy region from the threshold up to $w \approx 1.5 \mathrm{GeV}$. This remarkable dynamics of IPE distinguishes it essentially from the photoproduction and electroproduction, where rescattering effects amount $\approx 40-50 \%$.

Let us explain the quasithreshold behaviour of the IPE amplitude. As $\mathbf{k} \rightarrow 0$ the multipole amplitudes behave in the following way

$$
\begin{aligned}
& M_{l \pm} \propto \mathbf{k}^{l}, \quad E_{l+} \propto \mathbf{k}^{l}, \quad L_{l+} \propto \mathbf{k}^{l}, \\
& E_{l-} \propto \mathbf{k}^{l-2}, \quad L_{l-} \propto \mathbf{k}^{l-2} .
\end{aligned}
$$

Therefore, at $\mathbf{k}=0$ only the electric $\left(E_{0+}\right.$ and $\left.E_{2-}\right)$ and longitudinal $\left(L_{0+}\right.$ and $\left.L_{2-}\right)$ dipoles survive. In addition, the number of independent dipole transitions diminishes to the two ones at the quasithreshold due to the quasithreshold constraints

$$
E_{0+}=L_{0+}, \quad E_{2-}=-L_{2-},
$$

which arise from the causality (analyticity). The selection rules which follow from the parity conservation and the value of the angular momentum of the stopped virtual photon, $J=1$, restrict possible s-channel resonances at the quasithreshold to the following sets: $J^{P}=\frac{1}{2}^{-}\left[S_{11}(1535), S_{31}(1650), S_{11}(1700)\right.$, etc. $]$ and $J^{P}=\frac{3}{2}^{-}\left[D_{13}(1520), D_{33}(1670)\right.$, etc.]. Behaviour of the multipole amplitudes as $\mathbf{q} \rightarrow 0$ and $\mathbf{k} \rightarrow 0$ and the quasithreshold constraints among them are derived in Appendix B. 
Since the $s$ - and $d$-wave $\pi N$ resonances are excited at energies above $1.5 \mathrm{GeV}$, one can expect that the dipoles $E_{0+}$ and $E_{2-}$ are dominated by the Born terms below this energy. This is in agreement with the multipole analyses of charged pion photoproduction and confirmed by the dispersion-relation calculations at $\lambda^{2} \neq 0$. We can, therefore, conclude that in the quasithreshold region, the IPE amplitude is given by the Born terms with accuracy better than $5 \%$. At energies below $\approx 1.5 \mathrm{GeV}$, we can write for the quasithreshold IPE

$$
\begin{aligned}
\lim _{\mathbf{k} \rightarrow 0} \frac{\mathbf{q}}{\mathbf{k}} \frac{d^{2} \sigma}{d \lambda^{2} d \cos \theta} \approx \frac{\alpha}{12 \pi} \frac{m^{2}}{(\sqrt{s}-m)^{2}} \frac{1}{s}\left[\left(1+\cos ^{2} \theta\right)\left|E_{0+}^{\text {Born }}+E_{2-}^{\text {Born }}\right|^{2}+\right. \\
\left.+\sin ^{2} \theta\left|E_{0+}^{\text {Born }}-2 E_{2-}^{\text {Born }}\right|^{2}\right] .
\end{aligned}
$$

In the real experiment, however, one cannot realize strictly the quasithreshold conditions and, therefore, the realistic model (presented above) is needed. In choosing the optimal geometry of the experiment for deriving the form factors, the "compensation curves" [18] can help. The curves are defined as curves in the $(s, t)$ plane along which the differential cross section is given only by the Born terms. These curves can be constructed by comparing photoproduction experimental data with the Born cross section and employing the existence theorem for implicit functions (more details on the compensation effect is given in Appendix C).

The method of determining the electromagnetic form factors from low-energy IPE is, therefore, based on utilizing the quasithreshold theorem, the realistic dispersion-relation model, and the compensation curve. This method has been already used in the experiments on the nucleon and nuclei ${ }^{12} \mathrm{C}$ and ${ }^{7} \mathrm{Li}[20,21]$.

TABLE I: Electromagnetic form factors obtained in experiments on the nucleon. The virtual photon momentum $\lambda^{2}$ is given in units of the pion mass.

\begin{tabular}{ccccccccccc}
\hline$\lambda^{2}\left[m_{\pi}^{2}\right]$ & 2.77 & 2.98 & 3.44 & 3.75 & 4.00 & 4.47 & 4.52 & 5.28 & 5.75 & 6.11 \\
\hline$F_{1}^{v}$ & 0.96 & 0.93 & 1.16 & 1.04 & 1.14 & 1.22 & 1.13 & 1.20 & 1.32 & 1.36 \\
$F^{\pi}$ & 0.91 & 0.85 & 1.04 & 0.91 & 0.99 & 1.04 & 0.95 & 1.01 & 1.12 & 1.16 \\
Error & 0.10 & 0.09 & 0.10 & 0.08 & 0.16 & 0.10 & 0.09 & 0.09 & 0.10 & 0.08 \\
\hline
\end{tabular}

In Table [ we present values of the electromagnetic form factors obtained in experiments on the nucleon. In Tab. [ the same experimental errors are given for $F_{1}^{v}$ and $F^{\pi}$ because in this $\lambda^{2}$-range these form factors can be connected with each other via the spectral function by the relation $F_{1}^{v}\left(\lambda^{2}\right)-F^{\pi}\left(\lambda^{2}\right)=\triangle\left(\lambda^{2}\right)$. The quantity $\triangle\left(\lambda^{2}\right)$, taken from the dispersion calculations [39], possesses a significantly smaller theoretical uncertainty than the calculated quantities $F_{1}^{v}$ and $F^{\pi}$. This is caused by cancellation of terms with the large uncertainties in the spectral function of $\triangle\left(\lambda^{2}\right)$ which, therefore, is dominated by the one-nucleon exchange contribution in the region $4 m_{\pi}^{2} \leq \lambda^{2} \lesssim 20 m_{\pi}^{2}$. One can see that this result is rather model-independent. Then the same experimental errors can be given for $F_{1}^{v}$ and $F^{\pi}$. Of course, having high-statistics data for IPE one need not use the relation of $F_{1}^{v}$ with $F^{\pi}$ in extracting these quantities. The values of $F_{1}^{v}$ are quite consistent with the calculations of the nucleon electromagnetic structure in framework of the unitary and analytic vector-meson dominance model [40].

Let us look at the possibility of investigating the form factor $G_{M}^{*}\left(\lambda^{2}\right)$ of the $\gamma^{*} N N_{3 / 2}^{*}$ vertex at $\lambda^{2}>0$. Whereas a measurement of the differential cross section for the electroproduction with unpolarised electrons in the $\Delta(1232)\left(P_{33}\right)$ region at $\theta^{\gamma}$ out of the compensation curve allows one to extract information about the form factors of the $\gamma^{*} N N_{3 / 2}^{*}$ vertex, this approach is not sufficiently effective for IPE because the dominance of the Born mechanism which reaches $\approx 95 \%$ at the quasithreshold is also considerable at lower values of $\lambda^{2}$. It turns out that the analysis of asymmetry $P_{l}$ (10) in the dilepton production near the quasithreshold gives us that chance.

We consider the quantity $T_{5}$, connected to the asymmetry $P_{l}$. It can be expanded to series in $\mathbf{k}$ near the quasithreshold with taking into account formulas (11), (B1) and (B5)

$$
T_{5}=2 \frac{\alpha m^{2}}{\pi s} \sin \theta^{\gamma} \sum_{i=0} \mathbf{k}^{i} t_{i}
$$

The lowest term of the formal series in Eq. (24)

$$
t_{0}=3 \cos \theta^{\gamma}\left[-\operatorname{Im} E_{2-}^{*}\left(L_{2-}+L_{0+}\right)+\operatorname{Im} E_{0+}^{*} L_{2-}\right]_{\mathbf{k}=0},
$$

equals zero at the quasithreshold as it follows from the constraints (22). Behaviour of $T_{5}$ for $\mathbf{k} \rightarrow 0$ then is

$$
T_{5} \propto \mathbf{k} t_{1}
$$


where

$$
t_{1}=\operatorname{Im}\left[\left(\tilde{M}_{1+}-\tilde{M}_{1-}\right)\left(E_{0+}^{*}+E_{2-}^{*}\right)+\tilde{E}_{1+}\left(E_{2-}^{*}-5 E_{0+}^{*}\right)+\tilde{L}_{1-}\left(E_{0+}^{*}-2 E_{2-}^{*}\right)-g \tilde{E}_{3-} E_{2-}^{*}\right]_{\mathbf{k}=\mathbf{0}} .
$$

In the $P_{33}(1232)$ region, the amplitudes $E_{0+}$ and $E_{2-}$ are dominated by the Born terms, and the imaginary parts of form factors are negligible. Contributions of $E_{1+}$ and $L_{1-}$ amount to less than $15 \%$ of that of $M_{1+}$. Multipole amplitudes $M_{1-}$ and $L_{1-}$, related to the excitation of the $P_{11}(1470)$ resonance, generally have to be very small, as it is seen in analyses of photoproduction (especially on the neutron). Therefore, with a good accuracy, we obtain

$$
T_{5} \sim\left(E_{0+}^{\text {Born }}+E_{2-}^{\text {Born }}\right) \operatorname{Im} M_{1+} .
$$

Since the quasithreshold relations (B5) seem to be approximately realized in a rather wide interval in $\lambda^{2}$, the asymmetry $P_{l}$ has to be sensitive to $\operatorname{Im} M_{1+}$ in the $P_{33}(1232)$ region. The measurement of $P_{l}$ would, therefore, allow one to study quantitatively the assumption about the dominance of the magnetic dipole transition and to extract information on the form factor $G_{M}^{*}\left(\lambda^{2}\right)$ at $\lambda^{2}>0$. This is possible because the contribution of the background part of the amplitude to $T_{5}$ is considerably suppressed in the quasithreshold region, the background part reducing as $\mathbf{k}^{2}$ for $\mathbf{k}$ becoming zero.

\section{METHOD OF DETERMINING THE PSEUDOSCALAR FORM FACTOR OF NUCLEON FROM THE QUASITHRESHOLD IPE}

Now let us discuss another interesting possibility of investigating the weak nucleon structure related to the nucleon Gamow-Teller transition described by the matrix element

$$
\left\langle N\left(p_{2}\right)\left|A_{\mu}^{\alpha}\right| N\left(p_{1}\right)\right\rangle=\bar{u}\left(p_{2}\right) \frac{\tau^{\alpha}}{2}\left[\gamma_{\mu} G_{A}\left(\lambda^{2}\right)+k_{\mu} G_{P}\left(\lambda^{2}\right)\right] \gamma_{5} u\left(p_{1}\right),
$$

where $A_{\mu}^{\alpha}$ is the axial-vector current, and $G_{A}\left(\lambda^{2}\right)$ and $G_{P}\left(\lambda^{2}\right)$ are the axial and induced pseudoscalar form factors, respectively.

An alternative description of IPE-which utilizes the current commutators, PCAC, and completeness-allows one to derive a low-energy theorem at the threshold, $\vec{q}=0$ and $\lambda^{2} \rightarrow m_{\pi}^{2}$, related to the approximate chiral symmetry and $O\left(m_{\pi}^{2}\right)$ corrections. Minimization of the continuum contribution at the quasithreshold justifies this approach up to $w \approx 1.5 \mathrm{GeV}[13$ ] with the continuum corrections being practically the same as in the dispersion-relation description. Then, one obtains for the longitudinal part of the $\pi^{-} p \rightarrow \gamma^{*} n$ amplitude at the quasithreshold retaining only the leading terms in $\lambda^{2} / m^{2}, t / m^{2}[13$, such that

$$
\begin{aligned}
E_{0+}-2 E_{2-}= & \frac{\lambda}{2 m_{\pi}^{2} f_{\pi}} \sqrt{\frac{(w+m)^{2}-m_{\pi}^{2}}{m w}}\left\{D(t)-\left(1+\frac{\lambda}{2 m}\right) D\left(m_{\pi}^{2}-\lambda^{2}\right)+\right. \\
& \left.+\frac{m_{\pi}^{2}}{2 m}\left[G_{A}\left(m_{\pi}^{2}-\lambda^{2}\right)-\frac{t}{2 m} G_{P}\left(m_{\pi}^{2}-\lambda^{2}\right)\right]\right\}
\end{aligned}
$$

where the constant of the $\pi \rightarrow \mu+\nu_{\mu}$ decay $f_{\pi}$ is defined by $\left\langle 0\left|A_{\mu}(0)\right| \pi(q)\right\rangle=i f_{\pi} q_{\mu}, D(t)=-2 m G_{A}(t)+t G_{P}(t)$, and the quasithreshold values of the variables are

$$
w_{\text {q.thr. }}=m+\lambda, \quad t_{\text {q.thr. }}=\left(m_{\pi}^{2}-\lambda^{2}\right) \frac{m}{m+\lambda} .
$$

$G_{A}$ was measured in various experiments, first of all in $\nu n \rightarrow \mu^{-} p$ and $\bar{\nu} p \rightarrow \mu^{+} n$. It is reasonable to use first this result

$$
G_{A}(t)=G_{A}(0)\left(1-t / M_{A}^{2}\right)^{-2}, \quad G_{A}(0)=-1.25, \quad M_{A}=(0.96 \pm 0.03) \mathrm{GeV} .
$$

However, it is difficult to obtain reliable information on $G_{P}$ in these experiments since its contribution to cross sections is kinematically suppressed (it is multiplied by the lepton masses). In the $\mu$-capture and $\beta$-decay experiments, there is a kinematically restricted small range of $|t|, \approx 0-0.01(\mathrm{GeV} / \mathrm{c})^{2}$, in which the weak form factor can be determined, though with a large error. For example, its value extracted from measurements of the $\mu$-capture in hydrogen [41] is $G_{P}\left(-0.88 m_{\mu}^{2}\right)=-8.7 \pm 1.9 . G_{P}$ has also been measured in the capture of polarized muons by ${ }^{28} \mathrm{Si}$ nuclei 42$]$.

Equation (28) shows that the kinematic suppression of the $G_{P}$ contribution is absent when the IPE data at the quasithreshold are used for extracting $G_{P}$. In this way, the pseudoscalar form factor $G_{P}(t)$ can be determined in the 


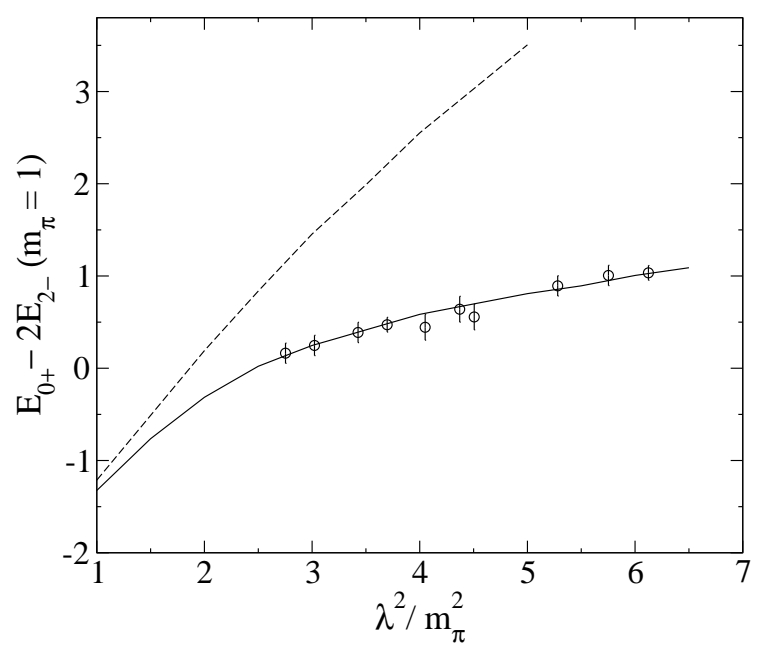

FIG. 4: Comparison of CA calculations for $E_{0+}-2 E_{2-}$ of the $\pi^{-} p \rightarrow \gamma^{*} n$ process with experimental data: dashed and solid curves correspond to the cases when $G_{P}$ is approximated by the pion pole $G_{P}^{\pi}$ and the prescription (31) is assumed, respectively. See the text for explanation of the data points.

range up to $t \approx-15 m_{\pi}^{2}$ (which corresponds to $w \approx 1.5 \mathrm{GeV}$ ). Adopting the quasithreshold kinematics, one can avoid the threshold difficulties that are present when applying an analogous method to the analysis of electroproduction data.

Next we shall follow the method of Ref. 15. First, using the $F_{1}^{v}\left(\lambda^{2}\right)$ and $F_{\pi}\left(\lambda^{2}\right)$ values obtained in the analysis of the IPE data on the nucleon [20], we obtain 10 data points for the longitudinal part of the $\pi^{-} p \rightarrow \gamma^{*} n$ amplitude at the quasithreshold. These data points, which can be considered as experimental ones, are depicted in Fig. 团

For $G_{P}(t)$ we chose the dispersion relation without subtractions:

$$
G_{P}(t)=\frac{2 f_{\pi} g_{\pi N}}{m_{\pi}^{2}-t}+\frac{1}{\pi} \int_{9 m_{\pi}^{2}}^{\infty} \frac{\rho\left(t^{\prime}\right)}{t^{\prime}-t} d t^{\prime}
$$

The residue at the pole $t=m_{\pi}^{2}$ is determined by the PCAC relation. When only the $\pi$-pole term is considered in $G_{P}$, the result is inconsistent with the experimental data as demonstrated in Fig. 团(dashed line). Therefore, the dispersion integral in Eq. (30) should be considered. It could be approximated by the contributions of possible intermediate three-pion and resonance states with the pion quantum numbers. However, since the contributions of nonresonant three-particle states must be suppressed by the phase volume, it is, therefore, reasonable to approximate the integral in (30) by a resonance-pole term. A satisfactory description is obtained if one takes the following expression for $G_{P}(t)$ with the indicated values of parameters:

$$
G_{P}(t)=G_{P}^{\pi}(t)-\frac{2 f_{\pi^{\prime}} g_{\pi^{\prime} N}}{m_{\pi^{\prime}}^{2}-t}, \quad 2 f_{\pi^{\prime}} g_{\pi^{\prime} N}=(1.97 \pm 0.18) \mathrm{GeV}, \quad m_{\pi^{\prime}}=0.5 \mathrm{GeV},
$$

where $G_{P}^{\pi}(t)=2 f_{\pi} g_{\pi N} /\left(m_{\pi}^{2}-t\right)$, the $\pi^{\prime}$ weak-decay constant $f_{\pi^{\prime}}$ is defined by $\left\langle 0\left|A_{\mu}(0)\right| \pi^{\prime}\left(q^{\prime}\right)\right\rangle=i f_{\pi^{\prime}} q_{\mu}^{\prime}$, and $g_{\pi N}(=13.5)$ and $g_{\pi^{\prime} N}$ are the coupling constants of the $\pi$ and $\pi^{\prime}$ states with the nucleon, respectively. As seen from the definitions of the weak-decay constants, one must expect that $f_{\pi^{\prime}} \ll f_{\pi}$ to reflect a tendency of another way (in addition to the Goldstone one) in which the axial current is conserved for vanishing quark masses. This behaviour is demonstrated in various models with some nonlocality which describe chiral symmetry breaking [43, 44]. Notice that the pole at $t=m_{\pi^{\prime}}^{2}$ in Eq. (31), situated considerably lower than the poles of the known contributing states $\pi^{\prime}(1300)$ and $\pi^{\prime}(1770)$, is indispensable for describing the obtained experimental data on IPE.

In Fig. [5 we show the ratio $G_{P}(t) / G_{P}^{\pi}(t)$ in comparison with the values obtained in $\mu$-capture on hydrogen 41. and in analysis of data on the $\pi^{+}$electroproduction off the proton near the threshold [45]. The error bars on the solid line indicate that the values of $G_{P}(t)$ determined by this method are of high accuracy. One can see that the results of Refs. [41, 45] agree with the pion-pole dominance hypothesis in a large range of momentum transfer unlike our result in which the hypothesis is valid only in a narrow $t$ range, whereas outside the range the contribution of continuum is considerable.

Note that the contributions of the pion radial excitations, $\pi^{\prime}(1300)$ and $\pi^{\prime}(1770)$, which are rather distant from this region, are suppressed and their consideration would only slightly increase the mass of $\pi^{\prime}(500)$. The parameters 


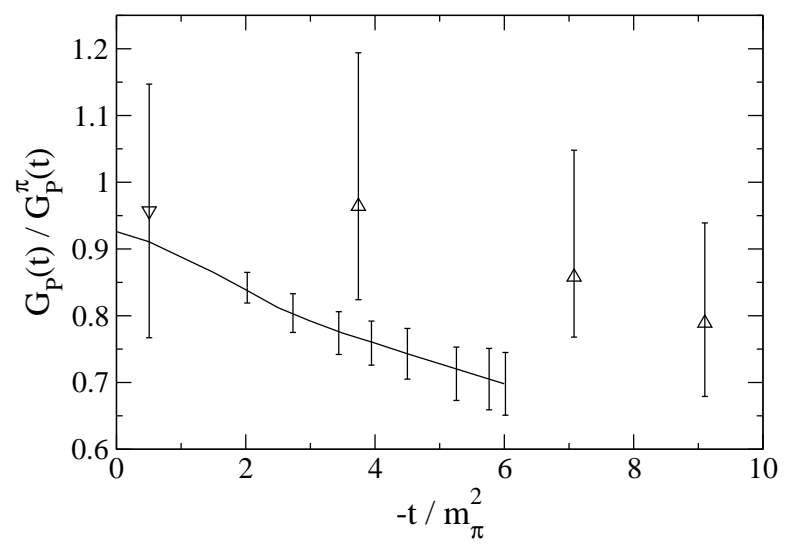

FIG. 5: Ratio $G_{P}(t) / G_{P}^{\pi}(t)$ as calculated with formula (31). The points on the curve with the error bar indicate the error corridor for this curve. Results of the data analysis on the $\mu$ capture in hydrogen $(\nabla)$ [41] and on the $\pi^{+}$electroproduction off the proton near the threshold $(\triangle)[45]$ are also depicted.

of this pole term in (31) might be changed more considerably if one assumes the channel $f_{0}(600) \pi$ with the recently discovered scalar $f_{0}(600)$ [6], due to the possible multichannel nature of this state. In any case, the conclusion will remain valid that the state with $I^{G}\left(J^{P}\right)=1^{-}\left(0^{-}\right)$in the range $500-800 \mathrm{MeV}$ is needed for explaining the obtained IPE data. Let us add that a possible signal of the charged state of this isotriplet was observed in the $\pi^{+} \pi^{-} \pi^{-}$system [47] and interpreted as the first radial excitation of the pion in the framework of the relativistic quark model [48] based on the covariant formalism for two-particle equations. Accepting this designation for $\pi^{\prime}(500-800)$ and taking the estimate for the $\pi^{\prime}$ weak-decay constant in the Nambu - Jona-Lasinio (NJL) model generalized by using effective quark interactions with a finite range, $f_{\pi^{\prime}}=0.65 \mathrm{MeV}$, we obtain $g_{\pi^{\prime} N}=1.51$. There are no suitable theoretical calculations for this coupling constant now. In the NJL model, the consideration of radial excitations of states requires introducing some nonlocality. Since a successful calculation of the $\pi N$ coupling constant in that model enforces one to go beyond the framework of the tree approximation and take loop corrections into account [49], it seems that a satisfactory evaluation of $g_{\pi^{\prime} N}$ in that approximation cannot avoid assuming some nonlocality. Of course, a more reliable interpretation of $\pi^{\prime}$ requires investigation of other processes with $\pi^{\prime}$. Existence of this state would also raise the question on its $\mathrm{SU}(3)$ partners. A careful (re)analysis of the corresponding processes is, therefore, desirable in this energy region.

\section{CONCLUSIONS}

We have demonstrated that a subsequent investigation of IPE is necessary for extracting both unique information about the electromagnetic structure of particles in the sub- $N \bar{N}$ threshold region of the timelike values of $\lambda^{2}$ and the nucleon weak structure in the spacelike region. The former is interesting especially now, e.g., in connection with discussion about the hidden strangeness of the nucleon (see, e.g., [50]) and quasinuclear bound $p \bar{p}$ state [51]. Analyses of the experimental IPE data in the first $\pi N$ resonance region allow one to obtain the values of the form factor $F_{1}^{v}$ at timelike values of $\lambda^{2}$ which are quite consistent with the calculations in the framework of the unitary analytic vectormeson dominance model 40. An inevitable step that is necessary for the study of the electromagnetic structure of nucleon-isobar systems in the timelike $\lambda^{2}$-region is a multipole analysis of IPE similar to that for the photoproduction and electroproduction, e.g. [23]. At present, with the intense pion beams being available, it is possible to perform experiments aiming at carrying out that analysis. In a construction of the dispersion-quark model in the second and third $\pi N$ resonance region, the multichannel character of the nucleon isobars must be taken into account, e.g., by the method of Ref. [52] or utilizing the proper uniforming variables [53].

As we already mentioned, our method was used in the analysis of the pion-induced dilepton production on the nucleon and light nuclei. It is worth making some remarks about the analysis of the experiment on the ${ }^{7} \mathrm{Li}$ nucleus with a $\pi^{+}$beam at $500 \mathrm{MeV} / \mathrm{c}$ [21]. The missing mass analysis of the data has shown that about half of the events are related to disintegration processes of the nucleus which are dominated by the reaction

$$
\pi^{+}+{ }^{7} \mathrm{Li} \rightarrow e^{+} e^{-}+p+{ }^{6} \mathrm{Li}
$$

On analyzing this process it was assumed that the pion-nucleus amplitude is determined by the neutron-pole mechanism, and the nuclear part (the vertex function of the ${ }^{7} \mathrm{Li} \rightarrow{ }^{6} \mathrm{Li}+n$ ) was calculated in the nucleon cluster model [54]. The remaining events belong to the process 


$$
\pi^{+}+{ }^{7} \mathrm{Li} \rightarrow e^{+} e^{-}+{ }^{7} \mathrm{Be} .
$$

When all the events were analyzed (with and without disintegration of the nucleus), the cross section on the nucleus was supposed to be additively connected with the cross section on the individual nucleon and nuclear effects were taken into account via screening. In both cases, our model was used for describing IPE on the individual nucleon. The obtained values for $F_{1}^{v}$ are again quite consistent with the calculations in Ref. [40]. In the case of the reaction without disintegration of the nucleus, one observed there the electromagnetic form factor of the nucleus in the timelike $\lambda^{2}$ region for the first time. Unfortunately, in the indicated analysis, unique information on the electromagnetic structure of the nucleus in the timelike region was lost. Generally, it seems at present that there is no satisfactory concept of the electromagnetic form factors of the nucleus in the timelike region. A satisfactory description must take into account both a constituent character of the nucleus (and the corresponding analytic properties) and more subtle (than the screening) collective nuclear effects.

Finally, it should be noticed that a more reliable interpretation of the observed state $\pi^{\prime}(500-800)$ requires solving a number of questions, both theoretical and experimental. In the pseudoscalar sector, states of various nature are possible, except for $q \bar{q}$, the $g g$ and $g g g$ glueballs, $q \bar{q} g$ hybrids, and multiquark states. However, all the models and lattice calculations give masses of those unusual states considerably greater than $1 \mathrm{GeV}$. Therefore, the most probable interpretation of $\pi^{\prime}(500-800)$ seems to be the first radial pion excitation.

\section{Acknowledgments}

The authors are grateful to S.B. Gerasimov, V.A. Meshcheryakov, and G.B. Pontecorvo for useful discussions and interest in this work. Yu.S. acknowledges also support provided by the Votruba-Blokhintsev Program for Theoretical Physics of the Committee for Cooperation of the Czech Republic with JINR, Dubna. P.B. thanks the Grant Agency of the Czech Republic, Grant No. 202/05/2142, and the Institutional Research Plan AVOZ10480505. M.N. acknowledges the Slovak Scientific Grant Agency, Grant VEGA No. 2/3105/23.

\section{APPENDIX A}

The invariant amplitudes $A_{i}\left(s, t, \lambda^{2}\right)$ relate to the scalar c.m. amplitudes $F_{i}\left(W, \cos \theta^{\gamma}, \lambda^{2}\right)$ as follows

$$
\begin{aligned}
& K A_{1}=\left(s-m^{2}-\lambda^{2}\right)\left(\frac{F_{1}}{p_{10}-m}-\frac{p_{20}+m}{\mathbf{q} \mathbf{k}} F_{2}\right)+\frac{m}{\mathbf{q} \mathbf{k}}\left[2 q_{0} \lambda^{2}+\right. \\
&\left.\left(t-m_{\pi}^{2}-\lambda^{2}\right) k_{0}\right]\left(\frac{F_{3}}{p_{10}-m}+\frac{p_{20}+m}{\mathbf{q k}} F_{4}\right)-2 m \lambda^{2}\left(\frac{F_{5}}{p_{10}-m}+\frac{p_{20}+m}{\mathbf{q k}} F_{6}\right), \\
& K A_{2}= \frac{2}{t-m_{\pi}^{2}-\lambda^{2}}\left\{\lambda^{2}\left(\frac{F_{1}}{p_{10}-m}-\frac{p_{20}+m}{\mathbf{q k}} F_{2}\right)+\frac{1}{2 \mathbf{q} \mathbf{k}}\left[\left(t-m_{\pi}^{2}-\lambda^{2}\right) k_{0}+\right.\right. \\
&\left.2 q_{0} \lambda^{2}\right]\left[(w-m) \frac{F_{3}}{p_{10}-m}-\frac{p_{20}+m}{\mathbf{q k}}(w+m) F_{4}\right]- \\
&\left.\lambda^{2}\left[(w-m) \frac{F_{5}}{p_{10}-m}-\frac{p_{20}+m}{\mathbf{q k}}(w+m) F_{6}\right]\right\} \\
& K A_{3}= K A_{4}+2 w \frac{\mathbf{k}}{\mathbf{q}}\left(\frac{F_{3}}{p_{10}-m}+\frac{p_{20}+m}{\mathbf{q k}} F_{4}\right), \\
& K A_{4}=(w-m) \frac{F_{1}}{p_{10}-m}+(w+m) \frac{p_{20}+m}{\mathbf{q k}} F_{2}+\frac{1}{2 \mathbf{q k}}\left[\left(t-m_{\pi}^{2}-\lambda^{2}\right) k_{0}+\right. \\
&\left.2 q_{0} \lambda^{2}\right]\left(\frac{F_{3}}{p_{10}-m}+\frac{p_{20}+m}{\mathbf{q k}} F_{4}\right)-\lambda^{2}\left[\frac{F_{5}}{p_{10}-m}+\frac{p_{20}+m}{\mathbf{q k}} F_{6}\right] \\
& \frac{2}{t-m_{\pi}^{2}-\lambda^{2}}\left\{\left(s-m^{2}\right)\left(\frac{-F_{1}}{p_{10}-m}+\frac{p_{20}+m}{\mathbf{q k}} F_{2}\right)+\frac{1}{2 \mathbf{q k}}\left[\left(t-m_{\pi}^{2}-\right.\right.\right. \\
&\left.\left.\lambda^{2}\right)\left(p_{10}+w\right)+2 q_{0}\left(s-m^{2}\right)\right]\left[(w-m) \frac{-F_{3}}{p_{10}-m}+\frac{p_{20}+m}{\mathbf{q k}}(w+m) F_{4}\right]+ \\
&\left.\left(s-m^{2}\right)\left[(w-m) \frac{F_{5}}{p_{10}-m}-\frac{p_{20}+m}{\mathbf{q k}}(w+m) F_{6}\right]\right\} \\
& A_{5}=
\end{aligned}
$$




$$
\begin{aligned}
K A_{6}= & (w+m) \frac{F_{1}}{p_{10}-m}+(w-m) \frac{p_{20}+m}{\mathbf{q k}} F_{2}-\frac{1}{2 \mathbf{q} \mathbf{k}}\left[\left(t-m_{\pi}^{2}-\lambda^{2}\right)\left(p_{10}+w\right)+\right. \\
& \left.2 q_{0}\left(s-m^{2}\right)\right]\left(\frac{F_{3}}{p_{10}-m}+\frac{p_{20}+m}{\mathbf{q} \mathbf{k}} F_{4}\right)-\left(s-m^{2}\right)\left(\frac{F_{5}}{p_{10}-m}+\frac{p_{20}+m}{\mathbf{q k}} F_{6}\right),
\end{aligned}
$$

where $K=\frac{2 s}{m} \sqrt{\left(p_{10}+m\right)\left(p_{20}+m\right)}$. The inverse relations reads

$$
\begin{aligned}
F_{1}= & \frac{\sqrt{\left((w+m)^{2}-\lambda^{2}\right)\left((w+m)^{2}-m_{\pi}^{2}\right)}}{4 m w}\left[(w-m) A_{1}+(w-m)^{2} A_{4}-\right. \\
& \left.\frac{1}{2}\left(t-m_{\pi}^{2}-\lambda^{2}\right)\left(A_{3}-A_{4}\right)-\lambda^{2} A_{6}\right], \\
F_{3}= & \sqrt{\left((w-m)^{2}-\lambda^{2}\right)\left((w-m)^{2}-m_{\pi}^{2}\right)} \frac{(w+m)^{2}-m_{\pi}^{2}}{8 m s}\left[\left(s-m^{2}\right) A_{2}+\right. \\
& \left.\lambda^{2} A_{5}+(w+m)\left(A_{3}-A_{4}\right)\right], \\
F_{5}= & \frac{1}{4 m w} \sqrt{\frac{(w+m)^{2}-m_{\pi}^{2}}{(w+m)^{2}-\lambda^{2}}}\left\{\frac { ( t - m _ { \pi } ^ { 2 } - \lambda ^ { 2 } ) } { 2 } \left[\left(3 s+m^{2}-\lambda^{2}\right) A_{2}+2 w\left(A_{3}-A_{4}\right)+\right.\right. \\
& \left.\left(s-m^{2}+\lambda^{2}\right) A_{5}\right]+\left[(w+m)^{2}-\lambda^{2}\right]\left[A_{1}+(w-m)\left(A_{4}-A_{6}\right)\right]+ \\
& \left.\left(s-m^{2}+m_{\pi}^{2}\right)\left[(w+m)\left(A_{3}-A_{4}\right)+\left(s-m^{2}\right) A_{2}+\lambda^{2} A_{5}\right]\right\} .
\end{aligned}
$$

Formulas for the remaining amplitudes can be obtained from Eqs. A22 using the formal substitution $w \rightarrow-w$ and symmetry properties of the amplitudes $F_{i}$ under the substitution

$$
F_{2}(w)=-F_{1}(-w), \quad F_{4}(w)=-F_{3}(-w), \quad F_{6}(w)=F_{5}(-w) .
$$

\section{APPENDIX B}

Here we demonstrate a derivation 29 of the behaviour of the multipole amplitudes as $\mathbf{q} \rightarrow 0$ and $\mathbf{k} \rightarrow 0$ on the basis of the first-class maximum analyticity principle [55]. According to this principle, the total amplitude possesses only singularities that are related to the dynamic processes and whose positions depend on masses of intermediate (and external) states which are involved in these processes. Then at the known arrangement of poles (and, therefore, of the threshold branch points) all the other singularities are determined by the systematic consideration of formulas for discontinuities in all channels.

Notice that the limit $\mathbf{k} \rightarrow 0$ is fulfilled in two cases: $p_{10} \rightarrow m$ and $p_{10} \rightarrow-m$. In the former case, the limit is fulfilled in IPE at the quasithreshold when the virtual photon has a maximal mass, whereas in the latter, the limit is fulfilled in the $k^{2}$-channel, $e^{+} e^{-} \rightarrow \pi N_{2} \bar{N}_{1}$, at the stopped antinucleon $\left(\bar{p}_{10}=m\right)$.

The amplitudes $F_{i}$ can be decomposed in terms of the multipole amplitudes: the magnetic $M_{l \pm}(j=l)$, electric $E_{l \pm}(j=l \pm 1)$, and longitudinal $L_{l \pm}(j=l \pm 1)$ (or scalar $S_{l \pm}$ but due to the current conservation, $\left.L_{l \pm}=\left(k_{0} / \mathbf{k}\right) S_{l \pm}\right)$ :

$$
\begin{aligned}
F_{1} & =\sum_{l=0}^{\infty}\left[l M_{l+}+E_{l+}\right] P_{l+1}^{\prime}\left(\cos \theta^{\gamma}\right)+\sum_{l=2}^{\infty}\left[(l+1) M_{l-}+E_{l-}\right] P_{l-1}^{\prime}\left(\cos \theta^{\gamma}\right), \\
F_{2} & =\sum_{l=1}^{\infty}\left[(l+1) M_{l+}+l M_{l-}\right] P_{l}^{\prime}\left(\cos \theta^{\gamma}\right), \\
F_{3} & =\sum_{l=1}^{\infty}\left[-M_{l+}+E_{l+}\right] P_{l+1}^{\prime \prime}\left(\cos \theta^{\gamma}\right)+\sum_{l=3}^{\infty}\left[M_{l-}+E_{l-}\right] P_{l-1}^{\prime \prime}\left(\cos \theta^{\gamma}\right), \\
F_{4} & =\sum_{l=2}^{\infty}\left[M_{l+}-M_{l-}-E_{l+}-E_{l-}\right] P_{l}^{\prime \prime}\left(\cos \theta^{\gamma}\right), \\
k_{0} F_{5} & =\sum_{l=0}^{\infty} L_{l+} P_{l+1}^{\prime}\left(\cos \theta^{\gamma}\right)-\sum_{l=2}^{\infty} L_{l-} P_{l-1}^{\prime}\left(\cos \theta^{\gamma}\right), \\
k_{0} F_{6} & =\sum_{l=1}^{\infty}\left[L_{l-}-L_{l+}\right] P_{l+1}^{\prime}\left(\cos \theta^{\gamma}\right) .
\end{aligned}
$$


Notice the unphysical multipoles: $M_{0+}=E_{0-}=L_{0-}=E_{1-}=0$. The multipole amplitudes are functions of $w=\sqrt{s}$ and $\lambda^{2}$ only. The properties of the amplitudes $F_{i}\left(w, \lambda^{2}, \cos \theta^{\gamma}\right)$ with respect to the substitution $w \rightarrow-w$ (A3) result in the corresponding symmetry relations for the multipole amplitudes

$$
\begin{aligned}
-(l+1) M_{l+}(-w) & =E_{(l+1)-}(w)+(l+2) M_{(l+1)-}(w), \\
(l+1) E_{l+}(-w) & =l E_{(l+1)-}(w)-M_{(l+1)-}(w), \\
L_{l+}(-w) & =-L_{(l+1)-}(w) .
\end{aligned}
$$

In the following, it is convenient to introduce new functions $\tilde{P}_{l}^{(n)}$ instead of the Legendre polynomial which have singularities (due to $\cos \theta^{\gamma}=\left(t-m_{\pi}^{2}-\lambda^{2}-2 q_{0} k_{0}\right) / 2 \mathbf{q k}$ )

$$
\tilde{P}_{l}=(\mathbf{q k})^{l} P_{l}, \quad \tilde{P}_{l}^{(n)}=(\mathbf{q k})^{l-n} P_{l}^{(n)} .
$$

Taking into account the relation between $F_{i}\left(w, \theta^{\gamma}, \lambda^{2}\right)$ and $A_{i}\left(s, t, \lambda^{2}\right)$, we can conclude that the quantities

$$
\begin{aligned}
& {\left[\left(p_{10}+m\right)\left(p_{20}+m\right)\right]^{-1 / 2} F_{1}, \quad\left[\left(p_{10}-m\right)\left(p_{20}-m\right)\right]^{-1 / 2} F_{2}, \quad \frac{\left[\left(p_{10}-m\right)\left(p_{20}-m\right)\right]^{-1 / 2}}{p_{20}+m} F_{3},} \\
& \frac{\left[\left(p_{10}+m\right)\left(p_{20}+m\right)\right]^{-1 / 2}}{p_{20}-m} F_{4}, \quad\left(\frac{p_{10}+m}{p_{20}+m}\right)^{1 / 2} F_{5}, \quad\left(\frac{p_{10}-m}{p_{20}-m}\right)^{1 / 2} F_{6}
\end{aligned}
$$

possess no kinematic singularities.

To guarantee this property of the quantities (B14) the multipole amplitudes in (B1) must have the form

$$
\begin{aligned}
& M_{l+}=\left(\frac{p_{20}+m}{p_{10}+m}\right)^{1 / 2}(\mathbf{q k})^{l} \tilde{M}_{l+}, \quad M_{l-}=\left[\left(p_{10}+m\right)\left(p_{20}+m\right)\right]^{-1 / 2}(\mathbf{q k})^{l} \tilde{M}_{l-}, \\
& E_{l+}=\left(\frac{p_{20}+m}{p_{10}+m}\right)^{1 / 2}(\mathbf{q k})^{l} \tilde{E}_{l+}, \quad E_{l-}=\left(\frac{p_{10}+m}{p_{20}+m}\right)^{1 / 2}(\mathbf{q})^{l} \mathbf{k}^{l-2} \tilde{E}_{l-}, \\
& L_{l+}=k_{0}\left(\frac{p_{20}+m}{p_{10}+m}\right)^{1 / 2}(\mathbf{q k})^{l} \tilde{L}_{l+}, \quad L_{l-}=k_{0}\left(\frac{p_{10}+m}{p_{20}+m}\right)^{1 / 2}(\mathbf{q})^{l} \mathbf{k}^{l-2} \tilde{L}_{l-}
\end{aligned}
$$

Furthermore, we must ensure that the expression

$$
\frac{\sum_{l=0}^{\infty}\left[l \tilde{M}_{l+}+\tilde{E}_{l+}\right] \tilde{P}_{l+1}^{\prime}}{p_{10}+m}
$$

in $F_{1}$ has no kinematic singularities at $p_{10}=-m$, i.e., we must demand the following constraint:

$$
l \tilde{M}_{l+}+\tilde{E}_{l+} \stackrel{p_{10 \rightarrow-}}{\longrightarrow}\left(p_{10}+m\right) \mu_{l} .
$$

In the exceptional case $l=0,\left(M_{0+} \equiv 0\right)$ we obtain

$$
\tilde{E}_{0+} \stackrel{p_{10 \rightarrow-m}}{\longrightarrow}\left(p_{10}+m\right) \mu_{0},
$$

i.e.,

$$
E_{0+}=\left[\left(p_{10}+m\right)\left(p_{20}+m\right)\right]^{1 / 2} \mu_{0} .
$$

It is clear that a question about the kinematic zeros in $F_{i}$ is left open. Since we are interested mainly in the $\lambda^{2}$ dependence, we give only the kinematic $\lambda^{2}$ behaviour of the amplitudes $F_{i}$ as $p_{10} \rightarrow m$ and $p_{10} \rightarrow-m$, not writing down explicitly the coefficients that depend only on $w$ at $\mathbf{k}=0$.

From (B1) and (B3)-(B8), we have

a) As $p_{10} \rightarrow m$

$$
F_{1} \sim F_{4} \sim F_{5} \propto 1, \quad F_{2} \sim F_{3} \propto\left(p_{10}-m\right)^{1 / 2}, \quad F_{6} \propto\left(p_{10}-m\right)^{-1 / 2}
$$

b) As $p_{10} \rightarrow-m$

$$
F_{1} \sim F_{4} \sim F_{5} \propto\left(p_{10}+m\right)^{1 / 2}, \quad F_{2} \sim F_{3} \sim F_{6} \propto 1, \quad F_{5} \propto\left(p_{10}+m\right)^{-1 / 2} .
$$


Taking into account formulas (A1), (Bי) and (B10), and the fact that the amplitudes $A_{i}$ have no kinematic singularities (except for the pole at $\lambda^{2}=t-m_{\pi}^{2}$ in $A_{2}$ and $A_{5}$ ), we conclude that the following constraints must be fulfilled to satisfy the maximal analyticity:

a) As $p_{10} \rightarrow m$

$$
\begin{aligned}
& F_{1}+\cos \theta^{\gamma} F_{4}-k_{0} F_{5} \propto\left(p_{10}-m\right), \\
& \cos \theta^{\gamma} F_{4}-k_{0} F_{6} \propto\left(p_{10}-m\right)^{1 / 2},
\end{aligned}
$$

b) As $p_{10} \rightarrow-m$

$$
\begin{aligned}
& F_{2}+\cos \theta^{\gamma} F_{4}-k_{0} F_{6} \propto\left(p_{10}+m\right), \\
& \cos \theta^{\gamma} F_{3}-k_{0} F_{5} \propto\left(p_{10}-m\right)^{1 / 2} .
\end{aligned}
$$

Constraints (B11)-(B14) and the $\lambda^{2}$ behaviour of the amplitudes $F_{i}$, (B9) and (B10), ensure the absence of kinematic singularities in the amplitude $F$, Eq. (5), as $p_{10} \rightarrow \pm m$ and $t=$ const.

Taking into account the constraints (B11)-(B14) and Eq. (B5) and using the recurrent formulas for the Legendre polynomials, we obtain kinematic relations between the multipole amplitudes at the quasithreshold and for $p_{10} \rightarrow-m$. The constraint (B12) gives as $p_{10} \rightarrow m$

$$
(l-1) \tilde{E}_{l-}+k_{0} \tilde{L}_{l-}=\left(p_{10}-m\right) \kappa_{l} \quad(l \geq 2),
$$

and

$$
L_{1-}=k_{0}\left[\left(p_{10}-m\right)\left(p_{20}-m\right)\right]^{1 / 2} \kappa_{1} .
$$

The constraint (B11) gives

$$
(l+1) \tilde{E}_{l+}-k_{0} \tilde{L}_{l+}=\left(p_{10}-m\right) \tau_{l},
$$

and (B14), as $p_{10} \rightarrow-m$, results in

$$
(l+1) \tilde{E}_{l+}-k_{0} \tilde{L}_{l+}=\left(p_{10}+m\right) \nu_{l} \quad(l \geq 1),
$$

or

$$
l(l+1) \tilde{M}_{l+}+k_{0} \tilde{L}_{l+}=\left(p_{10}+m\right)\left[(l+1) \mu_{l}-\nu_{l}\right] .
$$

The multipole amplitude $L_{0+}$, behaves as

$$
\tilde{L}_{0+}=k_{0}\left[\left(p_{10}+m\right)\left(p_{20}+m\right)\right]^{1 / 2} \nu_{0} .
$$

Finally, the constraint $(\mathbb{B 1 3})$ gives the following relations between the multipole amplitudes as $p_{10} \rightarrow-m$

$$
\begin{aligned}
\tilde{M}_{l-}-\frac{(l-1) \tilde{E}_{l-}+k_{0} \tilde{L}_{l-}}{p_{10}-m} & =\left(p_{10}+m\right) \rho_{l}, \quad(l \geq 2), \\
\tilde{M}_{1-}-k_{0} \kappa_{1} & =\left(p_{10}+m\right) \rho_{1} .
\end{aligned}
$$

So, we derived the constraints for the multipole amplitudes at the quasithreshold and as $p_{10} \rightarrow-m$. The relations of the first type (B15)-(B17) were also derived in Refs. 25, 38, 56, [57] by a different method that shades their kinematic nature. The relations for $p_{10} \rightarrow-m$, analogous to (B66), (BB18)-(B21), were also obtained in a different way in Ref. [57]. Note that since kinematic conditions as $p_{10} \rightarrow-m\left(\lambda^{2} \rightarrow(w+m)^{2}\right)$ are obtained from the quasithreshold kinematic configuration $p_{10} \rightarrow m\left(\lambda^{2} \rightarrow(w-m)^{2}\right)$ through the substitution $w \rightarrow-w$, constraints for the multipole amplitudes as $p_{10} \rightarrow-m$ can be derived from the ones at the quasithreshold with the help of the symmetry relations for multipole amplitudes (B2) taking into account the complete $\lambda^{2}$ behaviour of the latter as $p_{10} \rightarrow m$ (B5), (B15)-(B17). The relations for multipole amplitudes as $p_{10} \rightarrow-m$ are, of course, not fulfilled in the reaction under consideration. A region of their applicability is the c.m. system of pion and nucleon in the $\lambda^{2}$ channel $e^{+} e^{-} \rightarrow \pi N_{2} \bar{N}_{1}$ at the stopped antinucleon $\left(\bar{p}_{10} \rightarrow m\right)$.

The relations between multipole amplitudes at the quasithreshold are very important for our considerations because the condition $p_{10} \rightarrow m$ is realized (at $\lambda^{2}>m_{\pi}^{2}$ ) in the investigated reaction $\pi N \rightarrow e^{+} e^{-} N$ unlike the electroproduction and photoproduction. The introduced quantities $\kappa_{l}$ (B15) and $\tau_{l}$ (B17) are not formal coefficients of expansion in a series but they can be related to "toroid" multipole amplitudes $T_{l \pm}[38]$ that have a definite physical meaning. The relations $(\mathrm{B} 15)$ and $(\mathrm{B} 17)$ must be used with necessity when carrying out analytic continuations of spectral functions in the dispersion relations for IPE into the unphysical region $\left(m+m_{\pi}\right)^{2} \leq s^{\prime} \leq(m+\lambda)^{2}$. Therefore, it seems that application (from the beginning) of toroid multipoles $T_{l \pm}$ instead of $L_{l \pm}$ is rather relevant in the timelike region. 


\section{APPENDIX C}

Here we shall find a region of variables $s, t$, and $\lambda^{2}$ where the cross sections of the processes of pion photoproduction, electroproduction, and IPE are described only by the Born terms. Let us write the differential cross section of virtual photoproduction as a sum of two terms

$$
\frac{d \sigma}{d t}={\frac{d \sigma^{B o r n}}{d t}}^{\text {Bon }}+\Phi\left(s, t, \lambda^{2}\right),
$$

where the first term is the Born cross section and $\Phi\left(s, t, \lambda^{2}\right)$ takes into account the final-state interaction and its interference with the Born part of the amplitude.

To establish the conditions under which

$$
\Phi\left(s, t, \lambda^{2}\right)=0
$$

we use the existence theorem for implicit functions. By this theorem, if equation (C2) allows the solution $s=s_{0}$, $t=t_{0}, \lambda^{2}=\lambda_{0}^{2}$, and the function $\Phi\left(s, t, \lambda^{2}\right)$ and its partial derivatives of the first order are continuous in the vicinity of the point $M_{0}\left(s_{0}, t_{0}, \lambda_{0}^{2}\right)$ and the derivative $\Phi_{t}^{\prime}$ at this point is different from zero, that is,

$$
\Phi_{t}^{\prime}\left(s_{0}, t_{0}, \lambda_{0}^{2}\right) \neq 0,
$$

then there exists only one function

$$
t=f\left(s, \lambda^{2}\right),
$$

which satisfies equation (C2) in some vicinity of the point $M_{0}$ and takes the value $t=t_{0}$ at $s=s_{0}, \lambda^{2}=\lambda_{0}^{2}$. This function and its partial derivatives are continuous in the vicinity of the point $M_{0}$.

According to this theorem, if we find at least one point in the space of $\left(s, t, \lambda^{2}\right)$, where the effects of rescattering and their interference with the Born terms compensate each other, then, since the cross section is continuous in the physical region, there is a surface of "compensation" in this space on which the cross section is the Born one. The intersections of this surface with every plane $\lambda^{2}=$ const define some curves in the plane $(s, t)$ each of them being characterized by its own value of $\lambda^{2}$ and the cross section (C1) being the Born one along them. Thus, we obtain a one-parameter set of the compensation curves with $\lambda^{2}$ as a parameter.

Consider the curves $\lambda^{2}=0$ in more detail. We shall use $x=\cos \theta$ instead of variable $t$, where $\theta$ is a scattering angle in the c.m. system. If the compensation takes place, then in the plane $(x, s)$ we ought to have the compensation curve $x=f(s)$ continuous in the physical region. Generally speaking, there may be several curves of this kind, because the function $\Phi(s, x)$ can always be represented as a polynomial in powers of $x: \Phi(s, x)=\sum_{i} a_{i}(s) x^{i}$ and the equation $\sum_{i} a_{i}(s) x^{i}=0$ can have several real roots $x_{j}=f_{j}(s), j=1,2, \cdots$. However, due to the theorem mentioned above, the curves do not intersect, since only one curve can pass through the given point.
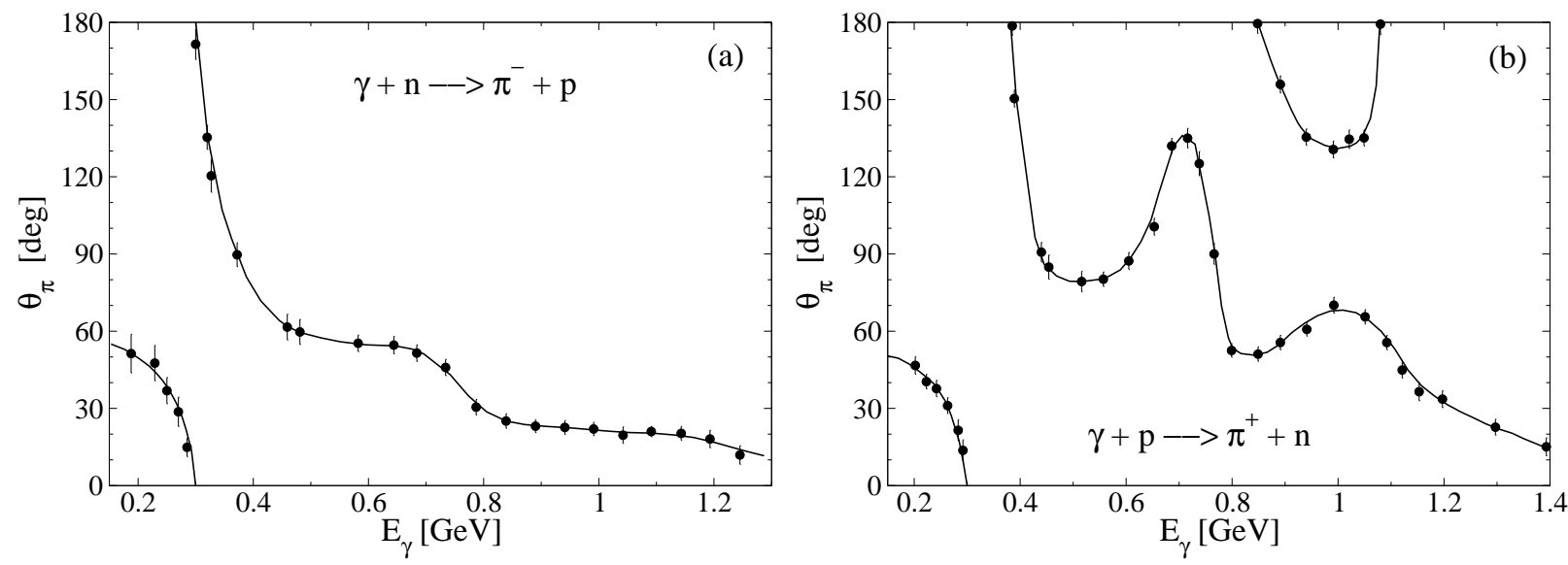

FIG. 6: Compensation curves for $\gamma p \rightarrow \pi^{+} n$ and $\gamma n \rightarrow \pi^{-} p$. Along these curves the differential cross section is described by EBM. At high energies, the compensation curve passes through the scattering angles corresponding to the value of the invariant momentum transfer $t \approx-0.04(\mathrm{GeV} / \mathrm{c})^{2}$. 
In the case of the pion photoproduction, available experimental data are sufficient to construct the compensation curves [18]. To this end, it is necessary to find out the intersection points of the experimental differential cross section with the calculated Born cross section and plot these points in the plane $(x, s)$ or $(\theta, s)$. In Fig. 6 the compensation curves for the charged pion photoproduction are shown. Calculating the Born cross sections, we take into account the interaction of the photon only with the charge. Therefore, along these curves the experimental cross sections are completely described by the electric Born terms. One can see that at high energies, the compensation curves pass through small angles in accordance with the electric Born model (EBM) [58, 59]. The compensation curves show also that an applicability of EBM to the cross section extends to the whole energy range, but at the corresponding angles, giving a more complete and transparent picture. Of course, there are also compensation curves corresponding to the total Born amplitude.

Now, if one assumes that for processes of the pion electroproduction and IPE, when $\lambda^{2} \neq 0$, the compensation curves are not much different from the corresponding curves for the photoproduction $\left(\lambda^{2}=0\right)$, then along these curves the model dependence of description of the processes should be minimal. In the resonance region, this assumption would be reasonable if the change of $\lambda^{2}$ does not lead to the essential reconstruction of the helicity (or multipole) structure of the excitation of the corresponding resonance. In the first resonance region, calculations, e.g., in the dispersion theory, which agree with the predictions of the quark models (e.g. [24, 28, 60]) and with the result of the phenomenological analysis of the electroproduction data (e.g. [57]), confirm a dominance preservation of the magnetic dipole excitation of the $P_{33}(1232)$ resonance with the change of $\lambda^{2}<0$. Quark models expand this situation to values of $\lambda^{2}>0$. In our dispersion model, it was verified that the compensation angle $\theta_{\text {comp }}^{\gamma}$ is invariable with $\lambda^{2}$ for the cross sections of the processes $\gamma^{*} n \leftrightarrow \pi^{-} p$ with the transverse virtual photons at $w=1.296 \mathrm{GeV}\left(\theta_{\text {comp }}^{\gamma} \simeq 70^{0}\right)$. This prediction was confirmed by the experiment 61] for the timelike values of $\lambda^{2}: \theta_{c o m p}^{\gamma}=70^{0} \pm 7^{0}$. In the second and third resonance regions, the analysis of experimental data for electroproduction (e.g. [57]) gives a considerable change (with $\left|\lambda^{2}\right|$ ) of the helicity structure for the excitation of leading resonances, which is especially essential approximately at $\lambda^{2} \simeq-1(\mathrm{GeV} / \mathrm{c})^{2}$.

The compensation curves exist also for the asymmetry in the charged pion production by the polarized $\gamma$-quanta

$$
\Sigma=\left[d \sigma_{\perp} / d t-d \sigma_{\|} / d t\right] /\left[d \sigma_{\perp} / d t+d \sigma_{\|} / d t\right] .
$$

For example, at $w=1.296 \mathrm{GeV}$ the compensation in $\Sigma$ (C5) takes place at $\theta_{\text {comp }}^{\gamma} \simeq 30^{0}$ for the $\pi^{ \pm}$photoproduction.

Since at high energies and small $|t|$ EBM (as any reasonable model explaining the sharp forward peak in $d \sigma / d t$ for the $\pi^{ \pm}$photoproduction) gives the sign and size of $\Sigma\left(\pi^{ \pm}\right)$in the forward direction, the compensation curves for $\Sigma\left(\pi^{ \pm}\right)$above the resonance region go through approximately the same values of the angles $\left(t \simeq 0.04(\mathrm{GeV} / \mathrm{c})^{2}\right)$ as for $d \sigma\left(\pi^{ \pm}\right) / d t$. This fact is explained practically model independently.

The plausibility of assumption that the compensation curves at high energies and small angles and at $\lambda^{2} \neq 0$ are not much different from the corresponding curves for $\lambda^{2}=0$ is justified by successful application of EBM to the description of the processes of electroproduction and $\pi N \rightarrow \rho^{0} N$ at high energies and $|t| \lesssim 2 m_{\pi}^{2}[58$, [59]. In any case, the compensation curves help one to reveal the optimal experimental conditions for studying the form factors $F_{\pi}\left(\lambda^{2}\right)$ and $F_{1}^{p}\left(\lambda^{2}\right)$ in processes of the pion electroproduction and IPE.

[1] E. Amaldi, S. Fubini, G. Furlan, Electroproduction at Low Energy and Hadron Form Factors, Springer Tracts in Modern Physics 83 (Springer, Berlin, 1979).

[2] Yu.S. Surovtsev, F.G. Tkebuchava, Yad. Fiz. 55, 2138 (1992).

[3] T.D. Blokhintseva, Yu.S. Surovtsev, M. Nagy, Acta Physica Slovaca 49, 351 (1999).

[4] A.I. Titov, B. Kämpfer, Eur. Phys. J. A 12, 217 (2001).

[5] E.R. Berger, M. Diehl, B. Pire, Phys. Lett. B 523, 265 (2001).

[6] M.F.M. Lutz, B. Friman, M. Soyeur, Nucl. Phys. A 713, 97 (2003).

[7] E.L. Bratkovskaya, W. Cassing, U. Mosel, Nucl. Phys. A 686, 568 (2001).

[8] D.A. Geffen, Phys. Rev. 125, 1745 (1962); J. Loubaton, J. Tran Thanh Van, Nucl. Phys. B 2, 342 (1967).

[9] Yu.S. Surovtsev, F.G. Tkebuchava, JINR Communication P2-4561 (Dubna, 1969); T.D. Blokhintseva, Yu.S. Surovtsev, F.G. Tkebuchava, Yad. Fiz. 21, 850 (1975).

[10] A.M. Baldin, V.A. Suleymanov, Phys. Lett. B 37, 305 (1971); JINR Communication P2-7096 (Dubna, 1973 ).

[11] Yu.S. Surovtsev, F.G. Tkebuchava, Yad. Fiz. 16, 1294 (1972).

[12] Yu.V. Kulish, Yad. Fiz. 16, 1102 (1972).

[13] G. Furlan, N. Paver, G. Verzegnassi, Nuovo Cimento A 32, 75 (1976).

[14] N. Dombey, B.S. Read, J. Phys. G 3, 1659 (1977).

[15] F.G. Tkebuchava, Nuovo Cimento A 47, 415 (1978).

[16] G.I. Smirnov, N.M. Shumeiko, Yad. Fiz. 17, 1266 (1973). 
[17] A. Bietti, S. Petrarca, Nuovo Cimento A 22, 595 (1974); Lett. Nuovo Cimento 13, 539 (1975).

[18] Yu.S. Surovtsev, F.G. Tkebuchava, Yad. Fiz. 21, 753 (1975).

[19] N.P. Samios, Phys. Rev. 121, 275 (1961); H. Kobrak, Nuovo Cimento 20, 1115 (1961); S. Devons et al., Phys. Rev. 184, 1356 (1969); M.N. Khachaturyan et al., Phys. Lett. B 24, 349 (1967); C.M. Hoffman et al., Phys. Rev. D 28, 660 (1983); H. Fonvieille et al., Phys. Lett. B 233, 60, 65 (1989).

[20] Yu.K. Akimov et al., Yad. Fiz. 13, 748 (1971); S.F. Berezhnev et al., ibid. 16, 185 (1972); 18, 102 (1973); 24, 1127 (1976); G.D. Alekseev et al., ibid. 36, 322 (1982); 46, 1360 (1987); V.V. Alizade et al., ibid. 33, 357 (1981).

[21] V.N. Baturin et al., Yad. Fiz. 47, 708 (1988).

[22] G. Bardin, Phys. Lett. B 255, 149 (1991); ibid. 257, 514 (1991).

[23] R.C.E. Devenish et al., Phys. Lett. B 52, 227 (1974); R.C.E. Devenish et al., Nuovo Cimento A 1, 475 (1971).

[24] S.A. Goghilidze, Yu.S. Surovtsev, F.G. Tkebuchava, Yad. Fiz. 45, 1085 (1987).

[25] S.L. Adler, Ann. Phys. (NY) 50, 189 (1968).

[26] R. Beck et al., Phys. Rev. C 61, 035204 (2000).

[27] Particle Data Group, D.E. Groom et al., Eur. Phys. J. C 15, 1 (2000).

[28] S. Capstick, Phys. Rev. D 46, 2864 (1992).

[29] Yu.S. Surovtsev, Author's summary, 2-11047, Dubna, 1977 (unpublished).

[30] I.G. Aznauryan, Phys. Rev. C 67, 015209 (2003).

[31] J. Volmer (JLab F $\pi$ Collaboration), Phys. Rev. Lett. 86, 1713 (2001).

[32] M.F. Gari and W. Kruempelmann, Phys. Rev. D 45, 1817 (1992).

[33] T. Fujii et al., Phys. Rev. Lett. 26, 1672 (1971); Nucl. Phys. B 120, 395 (1977); P. Benz et al., Nucl. Phys. B 65, 158 (1973); V. Rossi et al., Nuovo Cimento A 13, 59 (1973); M. Beneventano et al., Nuovo Cimento A 19, 529 (1974); G.von Holtey, G. Knop, H. Stein, J. Stümpfig, and H. Wahlen, Nucl. Phys. B 70, 379 (1974); P.E. Scheffler and P.L. Walden, Nucl. Phys. B 75, 125 (1974); A.J. Weiss et al., Nucl. Phys. B 101, 1 (1975); P.E. Argan et al., Nucl. Phys. A 296, 373 (1978); M.T. Tran et al., Nucl. Phys. A 324, 301 (1979); J.C. Comiso, D.J. Blasberg, R.P. Haddock, B.M.K. Nefkens, P. Truoel, and L.J. Verhey, Phys. Rev. D 12, 719 (1979); A. Bagheri, K.A. Aniol, F. Entezami, M.D. Hasinoff, D.F. Measday J-M. Poutissou, M. Salomon, and B.C. Robertson, Phys. Rev. C 38, 875 (1988); G.J. Kima, J. Arendsa, W.J. Briscoec, J. Engelagea, B.M.K. Nefkensa, M.E. Sadlerd, and H.J. Ziocka, Phys. Rev. D 40, 244 (1989).

[34] K. Bätzner et al., Phys. Lett. B 39, 575 (1972).

[35] G. Fischer et al., Z. Phys. 245, 225 (1971).

[36] G. Bardin, J. Duclos, A. Magnon, B. Michel and J.C. Montret, Nucl. Phys. B 120, 45 (1977).

[37] H. Breuker et al., Nucl. Phys. B 146, 285 (1978).

[38] V.M. Dubovik, L.A. Tosunyan, Fiz. Elem. Chastits. At. Yadra 14(5), 1193 (1983).

[39] G. Höhler and E. Pietarinen, Nucl. Phys. B 95, 210 (1975).

[40] S. Dubnička et al., Nuovo Cimento A 106, 1253 (1993).

[41] G. Bardin et al., Nucl. Phys. A 352, 365 (1981); Phys. Lett. B 104, 320 (1981).

[42] V. Brudanin et al., Nucl. Phys. A 587, 577 (1995).

[43] M.K. Volkov and C. Weiss, Phys. Rev. D 56, 221 (1997).

[44] Yu.L. Kalinovsky and C. Weiss, Z. Phys. C 63, 275 (1994); I.V. Amirkhanov et al., Modelling 6, 57 (1994).

[45] S. Choi et al., Phys. Rev. Lett. 71, 3927 (1993).

[46] K. Hagiwara et al. (Particle Data Group), Phys. Rev. D 66, 010001 (2002).

[47] Yu.I. Ivanshin et al., Nuovo Cimento A 107, 2855 (1994).

[48] Yu.I. Ivanshin, N.B. Skachkov, Nuovo Cimento A 108, 1263 (1995).

[49] A.N. Ivanov, M. Nagy, and N.I. Troitskaya, Phys. Rev. C 59, 451 (1999).

[50] S.B. Gerasimov, Chinese J. Phys. 34, 848 (1996).

[51] V.A. Meshcheryakov and G.V. Meshcheryakov, Yad. Fiz. 60, 1400 (1997).

[52] Guan-Yeu Chen, S.S. Kamalov, Shin Nan Yang, D. Drechsel, L. Tiator, Nucl. Phys. A 723, 447 (2003).

[53] D. Krupa, V.A. Meshcheryakov, Yu.S. Surovtsev, Nuovo Cimento A 109, 281 (1996).

[54] V.G. Avakov et al., Yad. Fiz. 44, 1471 (1986).

[55] G.F. Chew, S-Matrix Theory of Strong Interactions (Benjamin, New York, 1962).

[56] J.D. Bjorken and J.D. Walecka, Ann. Phys. (NY) 38, 35 (1966).

[57] R.C.E. Devenish and D.H. Lyth, Nucl. Phys. B 93, 109 (1975).

[58] N.N. Achasov and L.M. Samkov, Yad. Fiz. 8, 1201 (1968).

[59] C.F. Cho and J.J. Sakurai, Phys. Lett. B 30, 119 (1969).

[60] F.E. Close and F.J. Gilmal, Phys. Lett. B 38, 541 (1972).

[61] S.F. Berezhnev et al., Yad. Fiz. 26, 547 (1977). 